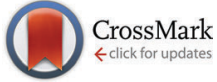

Cite this: New J. Chem., 2016, 40,5731

Received (in Montpellier, France) 13th November 2015. Accepted 22nd January 2016 DOI: $10.1039 / c 5 n j 03197 d$

www.rsc.org/njc

\title{
Synthesis and structural chemistry of bicyclic hexaaza-dithia macrocycles containing pendant donor groups $\dagger$
}

\author{
Mathias Gressenbuch, Ulrike Lehmann and Berthold Kersting*
}

\begin{abstract}
A short and efficient synthesis of a series of macrobicyclic aza-thioethers with pendant allyl $(\mathbf{8}, 13,14)$, cyanethyl (15), 3-aminopropyl (16), 2-methoxyacetyl (17, 19), 2-methoxyethyl (18, 20), and tertbutyloxycarbonyl substituents $(\mathbf{2 2}, \mathbf{2 3})$ has been achieved. The parent macrobicycles $\mathbf{1}$ and $\mathbf{2}$ are readily alkylated without overalkylation and without affecting the masked thiolate functions. The protocol is also feasible for the synthesis of macrobicycles with different alkyl groups on the benzylic and central nitrogen atoms of the linking diethylene triamine units. The identity of the compounds was substantiated using ESI MS, FT-IR, ${ }^{1} \mathrm{H}-\mathrm{NMR}$, and ${ }^{13} \mathrm{C}-\mathrm{NMR}$ spectroscopy. The crystal and molecular structures of six compounds $\left(8,15,17 \cdot 3 \mathrm{DMSO}, 19 \cdot 2 \mathrm{DMSO} \cdot 2 \mathrm{H}_{2} \mathrm{O}, 20\right.$ and 23) were additionally solved. The macrocycles are rather flexible and can adopt folded or stepped conformations. The ability of the compounds to form inclusion complexes with DMSO is also demonstrated. The crystal structures are governed by extensive inter- and intramolecular $\mathrm{CH} \cdots \pi$ interactions.
\end{abstract}

\section{Introduction}

The synthesis of macrocyclic ligands with pendant donor arms is highly desirable in view of a range of potential applications such as catalysis, selective cation binding, biomimetic chemistry, and radionuclide therapy. ${ }^{1-5}$ Thus, the chemistry of aliphatic polyaza-macrocycles has been well investigated as has the chemistry of their corresponding thia analogs. Many monocyclic macrocycles with side arms terminated with $\mathrm{N}, \mathrm{O}, \mathrm{S}$, or $\mathrm{P}$ donor groups have been prepared and their coordination chemistry investigated. ${ }^{6,7}$ Surprisingly, the chemistry of multidentate macrocycles with mixed $\mathrm{N}$ and $\mathrm{S}$ donor functions has received much less attention. ${ }^{8-10}$ This is true in particular for the families of amine-thioether ligands containing aromatic thioether groups. ${ }^{11-13}$ An early example is the 14-membered aromatic $\mathrm{N}_{2} \mathrm{~S}_{2}$ macrocycle, which was described by Lindoy and co-workers. ${ }^{14,15}$ Until now only few more ligands of this sort have been reported in the literature. ${ }^{16-23}$

Our group has reported the synthesis of the macrobicyclic azathioethers $\mathbf{1}^{24}$ and $2^{25}$ and of some alkylated derivatives 3-5 (Fig. 1). ${ }^{26,27}$ More recently, we have reported the first examples

Institut für Anorganische Chemie, Universität Leipzig, Johannisallee 29, D-04103 Leipzig, Germany.E-mail: b.kersting@uni-leipzig.de;

Fax: +49 (0)341-97-36199

$\dagger$ Electronic supplementary information (ESI) available: ${ }^{1} \mathrm{H},{ }^{13} \mathrm{C}$, IR and MS spectra for new compounds. Crystallographic data in CIF format for 8, 15, 17.3DMSO, 19. 2DMSO $2 \mathrm{H}_{2} \mathrm{O}, 20$ and 23. CCDC 1436363-1436368. For ESI and crystallographic data in CIF or other electronic format see DOI: 10.1039/c5nj03197d of bicyclic aminothioethers bearing pendant hydroxyethyl groups $(6,7){ }^{28}$ The structures of the free macrocycles $3,{ }^{29} 4,{ }^{25}$ $5^{, 27}$ and $\mathbf{6}^{28}$ have been determined. The macrocycles adopt a folded conformation in which the two aryl rings and the alkyl residues forming a cavity. However, none of these structures are clathrate, nor inclusion, complexes. As part of this program, we sought to extend our exploration to other variants of this versatile ligand system. To our knowledge, there are no systematic investigations on such hexaaza-thioether macrocycles. We have obtained a series of new macrobicyclic compounds bearing olefinic, nitrile, amine, and methoxy groups in place of the alkyl functions. Herein, we describe their synthesis and solid state structures. The effect of the pendant groups on the structural features is discussed, and compared with those of the parent ligand systems 1-3.

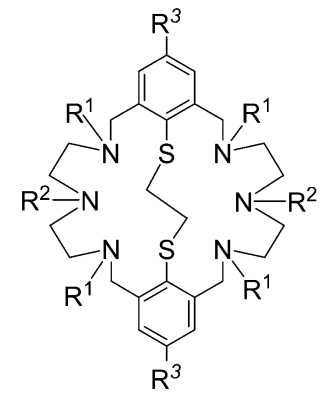

$1\left(R^{1}=R^{2}=H, R^{3}=t B u\right)$

$2\left(R^{1}=H, R^{2}=C_{3}, R^{3}=t B u\right)$

$3\left(R^{1}=R^{2}=\mathrm{CH}_{3}, R^{3}=t B u\right)$

$4\left(\mathrm{R}^{1}=\mathrm{CH}_{2} \mathrm{CH}_{3}, \mathrm{R}^{2}=\mathrm{CH}_{3}, \mathrm{R}^{3}=t \mathrm{Bu}\right)$

$5\left(R^{1}=R^{2}=\mathrm{CH}_{3}, \mathrm{R}^{3}=\mathrm{C}_{6} \mathrm{H}_{4}-\mathrm{tBu}\right)$

$6\left(\mathrm{R}^{1}=\mathrm{CH}_{2} \mathrm{CH}_{2} \mathrm{OH}, \mathrm{R}^{2}=\mathrm{CH}_{3}, \mathrm{R}^{3}=t \mathrm{Bu}\right)$

$7\left(\mathrm{R}^{1}=\mathrm{R}^{2}=\mathrm{CH}_{2} \mathrm{CH}_{2} \mathrm{OH}, \mathrm{R}^{3}=t \mathrm{Bu}\right)$

Fig. 1 Formula of macrobicyclic aza-thioethers 1-7. 


\section{Experimental section}

\section{Materials and physical measurements}

The bicyclic aza-thioethers $\mathbf{1}^{24}$ and $2,^{25}$ 1,2-bis(4-tert-butyl-2,6diformylthioethane) (10), ${ }^{24}$ bis(2-phthalimidoethyl)amine ${ }^{30}$ (11), and tert-butyl-bis(2-aminoethyl)carbamate $(\mathbf{2 1})^{31}$ were prepared according to literature. Melting points were determined with an Electrothermal IA9000 series instrument using open glass capillaries and are uncorrected. Mass spectra were obtained using the positive ion electrospray ionization modus (ESI) on a FT-ICR-MS Bruker Daltronics APEX II instrument. NMR spectra were recorded on a Bruker DRX-600, Bruker DRX-400 or Varian Mercury plus 400 spectrometer. Chemical shifts refer to solvent signals. The atom labels used to assign the NMR signals are not identical with those used in the X-ray structures. Elemental analyses were carried out with a VARIO EL - elemental analyzer.

\section{Synthesis and analysis of compounds}

The corresponding spectra for the IR, ${ }^{1} \mathrm{H}-\mathrm{NMR},{ }^{13} \mathrm{C}-\mathrm{NMR}$ are included in the ESI, $\dagger$ for each of the synthetized compounds. A summary of the obtained results are shown here.

Hexaallylated aza-thioether 8. The thioether 1 (307 mg, $0.50 \mathrm{mmol}$ ) and allyl bromide $(372 \mathrm{mg}, 3.07 \mathrm{mmol}$ ) were dissolved in EtOH (3 mL). A solution of triethylamine (303 mg, $3.00 \mathrm{mmol})$ in EtOH $(1 \mathrm{~mL})$ was added dropwise and the mixture was allowed to stand for 2 weeks at $0{ }^{\circ} \mathrm{C}$. The mixture was decanted off from the resulting $\mathrm{HNEt}_{3} \mathrm{Br}$. Crystals of the title compound crystallized from the mother liquor upon standing in air. Yield: $392 \mathrm{mg}$ (0.46 mmol, 92\%), colorless solid. M.p. 170-172 ${ }^{\circ} \mathrm{C}$. Found: $\mathrm{C} 72.90, \mathrm{H} 9.32, \mathrm{~N} 9.84, \mathrm{~S} 7.21 ; \mathrm{C}_{52} \mathrm{H}_{80} \mathrm{~N}_{6} \mathrm{~S}_{2}$ (853.37) requires: $\mathrm{C}$ 73.19, $\mathrm{H}$ 9.45, $\mathrm{N}$ 9.85, $\mathrm{S}$ 7.51. $\mathrm{m} / \mathrm{z}$ (ESI+, $\mathrm{MeOH}): \mathrm{C}_{52} \mathrm{H}_{80} \mathrm{~N}_{6} \mathrm{~S}_{2}$ (852.59) $\left[\mathrm{M}+\mathrm{H}^{+}\right]^{+}$calcd: 853.60; found 853.60. IR (KBr): $\nu / \mathrm{cm}^{-1}=3443 \mathrm{w}, 3078 \mathrm{w}, 3004 \mathrm{w}, 2962 \mathrm{vs}$, 2927 m, 2904 m, 2867 m, 2810 vs, 2712 vw, 1843 vw, 1643 w, 1593 w, 1559 vw 1477 w, 1459 w, 1442 w, 1418 w, 1404 w, 1358 m, 1260 m, 1242 w, 1229 w, 1153 w, 1100 s, 1023 vw, 993 m, 963 w, $917 \mathrm{vs}, 900 \mathrm{vw}, 863 \mathrm{vw}, 801 \mathrm{vw}, 734 \mathrm{vw}, 690 \mathrm{vw}, 639 \mathrm{vw}, 600 \mathrm{vw}$, 573 vw. ${ }^{1} \mathrm{H}-\mathrm{NMR}\left(400 \mathrm{MHz}, \mathrm{CDCl}_{3}\right): \delta=1.31\left(\mathrm{~s}, 18 \mathrm{H}, \mathrm{C}\left(\mathrm{CH}_{3}\right)_{3}\right)$, $2.60\left(\mathrm{~m}, 16 \mathrm{H},\left(\mathrm{NCH}_{2} \mathrm{CH}_{2} \mathrm{~N}\right)\right), 2.74\left(\mathrm{~s}, 4 \mathrm{H}, \mathrm{SCH}_{2}\right), 2.97(\mathrm{~d}, 8 \mathrm{H}$, $\left.{ }^{3} J=6.4 \mathrm{~Hz}, \mathrm{NCH}_{2} \mathrm{CHCH}_{2}\right), 3.10\left(\mathrm{~d}, 4 \mathrm{H},{ }^{3} \mathrm{~J}=6.4 \mathrm{~Hz}, \mathrm{NCH}_{2} \mathrm{CHCH}_{2}\right.$ ), $3.70\left(\mathrm{~s}, 8 \mathrm{H}, \mathrm{ArCH}_{2}\right), 5.05-5.18\left(\mathrm{~m}, 12 \mathrm{H}, \mathrm{CH}=\mathrm{CH}_{2}\right), 5.82(\mathrm{~m}, 6 \mathrm{H}$, $\left.\mathrm{CH}=\mathrm{CH}_{2}\right), 7.50(\mathrm{~s}, 4 \mathrm{H}, \mathrm{ArH}) .{ }^{13} \mathrm{C}\left\{{ }^{1} \mathrm{H}\right\}-\mathrm{NMR}\left(100 \mathrm{MHz}, \mathrm{CDCl}_{3}\right)$ : $\delta=31.60\left(\mathrm{C}\left(\mathrm{CH}_{3}\right)_{3}\right), \quad 34.98\left(C\left(\mathrm{CH}_{3}\right)_{3}\right), \quad 35.82\left(\mathrm{SCH}_{2}\right)_{2}, \quad 50.89$ $\left(\left(\mathrm{NCH}_{2} \mathrm{CH}_{2}\right)_{2} \mathrm{~N}\right), 51.71\left(\left(\mathrm{NCH}_{2} \mathrm{CH}_{2}\right)_{2} \mathrm{~N}\right), 56.88\left(\mathrm{ArCH}_{2} \mathrm{NCH}_{2} \mathrm{CHCH}_{2}\right)$, $57.51\left(\mathrm{ArCH}_{2}\right), 59.23\left(\mathrm{NCH}_{2} \mathrm{CHCH}_{2}\right), 116.99\left(\mathrm{ArCH}_{2} \mathrm{NCH}_{2} \mathrm{CH}=\right.$ $\left.\mathrm{CH}_{2}\right), 117.46\left(\mathrm{NCH}_{2} \mathrm{CH}=\mathrm{CH}_{2}\right), 124.86\left(\mathrm{ArC}-3,3^{\prime}\right), 128.18\left(\mathrm{ArC}-2,2^{\prime}\right)$, 136.35 $\left(\mathrm{ArCH}_{2} \mathrm{NCH}_{2} \mathrm{CH}=\mathrm{CH}_{2}\right), 136.34\left(\mathrm{CH}=\mathrm{CH}_{2}\right), 143.71(\mathrm{ArC}-1)$, 151.40 (ArC-4). This compound was additionally characterized by X-ray crystallography.

Tetraallylated aza-thioether 9. The thioether 2 (641 mg, $1.00 \mathrm{mmol}$ ) and allyl bromide (509 $\mathrm{mg}, 4.21 \mathrm{mmol}$ ) were dissolved in EtOH (3 mL). A solution of triethylamine (404 mg, $4.00 \mathrm{mmol})$ in $\mathrm{EtOH}(1 \mathrm{~mL})$ was added dropwise and the mixture was allowed to stand for 2 weeks at $0{ }^{\circ} \mathrm{C}$. $\mathrm{HNEt}_{3} \mathrm{Br}$ crystallized, from which the reaction mixture was decanted off. The title compound precipitated from the mother liquor upon standing in air.
This material was further purified by recrystallization from $\mathrm{MeCN} / \mathrm{CH}_{2} \mathrm{Cl}_{2}$. Yield: $649 \mathrm{mg}$ (0.46 mmol, 81\%), colorless solid. Found: $\mathrm{C}$ 72.0, $\mathrm{H}$ 9.42, $\mathrm{N}$ 9.94, $\mathrm{S}$ 7.81; $\mathrm{C}_{48} \mathrm{H}_{76} \mathrm{~N}_{6} \mathrm{~S}_{2}$ (801.30) requires: $\mathrm{C} 71.95, \mathrm{H}$ 9.56, $\mathrm{N}$ 10.49, $\mathrm{S}$ 8.00. $\mathrm{m} / \mathrm{z}(\mathrm{ESI}+, \mathrm{MeOH})$ : $\mathrm{C}_{48} \mathrm{H}_{76} \mathrm{~N}_{6} \mathrm{~S}_{2}$ (800.56) $\left[\mathrm{M}+\mathrm{H}^{+}\right]^{+}$calcd: 801.57; found 801.60. IR (KBr): $\nu / \mathrm{cm}^{-1}=3425 \mathrm{w}, 3072 \mathrm{w}, 3004 \mathrm{w}, 2962 \mathrm{vs,}, 2927 \mathrm{~m}, 2907 \mathrm{~s}$, $2867 \mathrm{~m}, 2810 \mathrm{~s}, 2787 \mathrm{~s}, 2703 \mathrm{~m}, 1829 \mathrm{vw}, 1641 \mathrm{w}, 1594 \mathrm{w}$, 1559 vw 1477 m, 1459 s, 1415 m, 1357 s, 1330 m, 1311 m, 1262 vs, 1216 w, 1194 w, 1154 s, 1106 vs, 1035 vs, 992 s, 980 m, 914 s, $883 \mathrm{~m}, 801$ vs, $741 \mathrm{vw}, 687$ w. ${ }^{1} \mathrm{H}-\mathrm{NMR}\left(400 \mathrm{MHz}, \mathrm{CDCl}_{3}\right)$ : $\delta=1.21\left(\mathrm{~s}, 18 \mathrm{H}, \mathrm{C}\left(\mathrm{CH}_{3}\right)_{3}\right), 2.21\left(\mathrm{~s} .6 \mathrm{H}, \mathrm{CH}_{3}\right), 2.50-2.59(\mathrm{~m}$, $\left.16 \mathrm{H}, \mathrm{NCH}_{2} \mathrm{CH}_{2} \mathrm{~N}\right), 2.85\left(\mathrm{~m}, 8 \mathrm{H}, \mathrm{NCH}_{2} \mathrm{CHCH}_{2}\right), 2.87(\mathrm{~s}, 4 \mathrm{H}$, $\mathrm{SCH}_{2}$ ), $3.76\left(\mathrm{~s}, 8 \mathrm{H}, \mathrm{ArCH}_{2}\right), 5.02-5.10\left(\mathrm{~m}, 8 \mathrm{H}, \mathrm{NCH}_{2} \mathrm{CHCH}_{2}\right.$ ), $5.74\left(\mathrm{~m}, 4 \mathrm{H}, \mathrm{CH}=\mathrm{CH}_{2}\right), 7.37(\mathrm{~s}, 4 \mathrm{H}, \mathrm{Ar} H) .{ }^{13} \mathrm{C}\left\{{ }^{1} \mathrm{H}\right\}-\mathrm{NMR}$ $\left(100 \mathrm{MHz}, \mathrm{CDCl}_{3}\right): \delta=31.39\left(\mathrm{C}\left(\mathrm{CH}_{3}\right)_{3}\right), 34.76\left(\mathrm{C}\left(\mathrm{CH}_{3}\right)_{3}\right)$, $36.69\left(\mathrm{SCH}_{2}\right)_{2}, \quad 42.94\left(\mathrm{CH}_{3}\right), \quad 51.16\left(\left(\mathrm{NCH}_{2} \mathrm{CH}_{2}\right)_{2} \mathrm{~N}\right), \quad 55.17$ $\left(\left(\mathrm{NCH}_{2} \mathrm{CH}_{2}\right)_{2} \mathrm{~N}\right), 56.58\left(\mathrm{NCH}_{2} \mathrm{CH}=\mathrm{CH}_{2}\right), 57.91\left(\mathrm{ArCH}_{2}\right), 117.50$ $\left(\mathrm{CH}=\mathrm{CH}_{2}\right), \quad 125.73 \quad\left(\mathrm{ArC}-3,3^{\prime}\right), \quad 129.31 \quad\left(\mathrm{ArC}-2,2^{\prime}\right), \quad 135.42$ $\left(\mathrm{CH}=\mathrm{CH}_{2}\right), 143.78(\mathrm{ArC}-1), 151.24(\mathrm{ArC}-4)$. This compound was additionally characterized by X-ray crystallography.

$\boldsymbol{N}$-Allyl-bis(2-phthalimidoethyl)-amine (12). A mixture of bis(2phthalimidoethyl)-amine $11(25.0 \mathrm{~g}, 68.8 \mathrm{mmol}), \mathrm{K}_{2} \mathrm{CO}_{3}(9.51 \mathrm{~g}$, $68.8 \mathrm{mmol})$, and allyl bromide $(11.6 \mathrm{~g}, 9.59 \mathrm{mmol})$ in $700 \mathrm{~mL}$ of THF was stirred for $30 \mathrm{~min}$ at room temperature and for $12 \mathrm{~h}$ at $50{ }^{\circ} \mathrm{C}$. The resulting mixture was filtered, and concentrated in vacuum to one fourth of its original volume. The resulting crystals were collected and dried under vacuum. Yield: $15.5 \mathrm{~g}, 56 \%, \mathrm{mp}$ $132{ }^{\circ} \mathrm{C}$. Found: $\mathrm{C}$ 68.19, $\mathrm{H}$ 5.54, N 10.34; $\mathrm{C}_{23} \mathrm{H}_{21} \mathrm{~N}_{3} \mathrm{O}_{4}$ (403.44) requires: $\mathrm{C}$ 68.47, $\mathrm{H} 5.25, \mathrm{~N} 10.42$. IR $(\mathrm{KBr}): \nu / \mathrm{cm}^{-1}=3461 \mathrm{w}$, 3091 vw, 3018 vw, 2955 w, 2835 w, 1769 s, 1711 vs, 1611 m, 1468 s, 1438 s, 1404 s, 1387 vs, 1332 m, 1306 m, 1276 w, 1191 w, 1160 vw, 1142 w, 1088 m,1041 m, 1018 s, 974 w, 939 w, 874 w, 803 w, 773 vw, 726 vs, 630 w, 613 w, 567 vw, 532 m, 469 vw. ${ }^{1} \mathrm{H}-\mathrm{NMR}(400 \mathrm{MHz}$, $\left.\mathrm{CDCl}_{3}\right): \delta=2.74\left(\mathrm{t},{ }^{3} J=6.4 \mathrm{~Hz}, 4 \mathrm{H},\left(\mathrm{NCH}_{2} \mathrm{CH}_{2}\right)_{2} \mathrm{~N}, 3.15(\mathrm{~d}\right.$, $\left.{ }^{3} J=6.4 \mathrm{~Hz}, 2 \mathrm{H}, \mathrm{CH}_{2} \mathrm{CH}=\mathrm{CH}_{2}\right), 3.67\left(\mathrm{t},{ }^{3} \mathrm{~J}=6.4 \mathrm{~Hz}, 4 \mathrm{H}\right.$, $\left(\mathrm{NCH}_{2} \mathrm{CH}_{2}\right)_{2} \mathrm{~N}, 4.98-5.08\left(\mathrm{dd}, 1 \mathrm{H},{ }^{3} \mathrm{~J}=16.4 \mathrm{~Hz},{ }^{3} \mathrm{~J}=10.4 \mathrm{~Hz}\right.$, $\left.\mathrm{CH}=\mathrm{CH}_{2}\right), 5.56-5.63\left(\mathrm{~m}, 2 \mathrm{H}, \mathrm{CH}=\mathrm{CH}_{2}\right), 7.60-7.67(\mathrm{~m}, 8 \mathrm{H}, \mathrm{ArH})$. $\left.{ }^{13} \mathrm{C}\left\{{ }^{1} \mathrm{H}\right\}-\mathrm{NMR}\left(100 \mathrm{MHz}, \mathrm{CDCl}_{3}\right): \delta=36.30\left(\mathrm{NCH}_{2} \mathrm{CH}_{2}\right)_{2} \mathrm{~N}\right)$, $51.90\left(\mathrm{NCH}_{2} \mathrm{CH}_{2}\right)_{2} \mathrm{~N}, 57.03\left(\mathrm{CH}_{2} \mathrm{CH}=\mathrm{CH}_{2}\right), 118.47\left(\mathrm{CH}=\mathrm{CH}_{2}\right)$, 123.59 (ArC-2,2' $)$ 132.73 (ArC-1,1' $), 133.96$ (ArC-3,3'), 135.56 $\left(\mathrm{CH}=\mathrm{CH}_{2}\right), 168.74(\mathrm{CO})$.

$\mathrm{N}$-Allyl-bis(2-aminoethyl)-amine trihydrochloride (13-3HCl). A suspension of $N$-allyl-bis(2-phthalimidoethyl)-amine (15.6 g, $38.7 \mathrm{mmol}$ ) was dissolved in $200 \mathrm{~mL}$ of concentrated $\mathrm{HCl}$ and refluxed for 2 days. The clear solution was cooled and filtered. The clear solution was concentrated in vacuum, to give a brown oil, which was washed with THF. The oil was separated and dried under vacuum to give a colorless, hygroscopic solid. The compound could not be obtained in analytically pure form but was found pure enough for the next step. Yield: $8.16 \mathrm{~g}$ (83\%). IR (KBr): $\nu / \mathrm{cm}^{-1}=3415 \mathrm{~m}, 2963 \mathrm{vs}, 2039 \mathrm{w}, 1602 \mathrm{~m}$, 1471 s, 1372 m, 1262 s, 1099 vs, 1019 vs, 959 s, 869 w, 801 vs, $662 \mathrm{vw}, 603 \mathrm{vw}, 461$ w. ${ }^{1} \mathrm{H}-\mathrm{NMR}\left(400 \mathrm{MHz}, \mathrm{D}_{2} \mathrm{O}\right): \delta=3.45$ $\left(\mathrm{m},{ }^{3} J=6.4 \mathrm{~Hz}, 4 \mathrm{H},\left(\mathrm{H}_{2} \mathrm{NCH}_{2}\right), 3.52\left(\mathrm{t},{ }^{3} \mathrm{~J}=6.4 \mathrm{~Hz}, 4 \mathrm{H},\left(\mathrm{CH}_{2}\right)_{2} \mathrm{~N}\right)\right.$, $3.89\left(\mathrm{~d},{ }^{3} \mathrm{~J}=7.2 \mathrm{~Hz}, 2 \mathrm{H}, \mathrm{NCH}_{2} \mathrm{CH}=\mathrm{CH}_{2}\right), 5.67(\mathrm{~m}, 1 \mathrm{H}$, $\left.\mathrm{CH}=\mathrm{CH}_{2}\right), 5.93\left(\mathrm{~m}, 2 \mathrm{H}, \mathrm{CH}=\mathrm{CH}_{2}\right) .{ }^{13} \mathrm{C}\left\{{ }^{1} \mathrm{H}\right\}-\mathrm{NMR}(100 \mathrm{MHz}$, 
$\left.\mathrm{D}_{2} \mathrm{O}\right): \delta=34.0\left(\mathrm{H}_{2} \mathrm{NCH}_{2}\right), 49.6\left(\left(\mathrm{CH}_{2}\right)_{2} \mathrm{~N}\right), 56.3\left(\mathrm{NCH}_{2}\right), 124.8$ $\left(\mathrm{CH}=\mathrm{CH}_{2}\right), 128.2\left(\mathrm{CH}=\mathrm{CH}_{2}\right)$.

$\mathrm{N}$-Allyl-bis(2-aminoethyl)-amin (13). A suspension of $\mathrm{N}$-allylbis(2-aminoethyl)-amine-trihydrochloride $(9.76 \mathrm{~g}, 38.6 \mathrm{mmol})$ and $\mathrm{KOC}\left(\mathrm{CH}_{3}\right)_{3}(13.0 \mathrm{~g}, 116 \mathrm{mmol})$ in $50 \mathrm{~mL}$ of THF was stirred at $55{ }^{\circ} \mathrm{C}$ for $3 \mathrm{~d}$ and filtered. The THF was removed in vacuum to give an oil, which was purified by distillation in vacuum. Yield: $5.25 \mathrm{~g}(89 \%)$. The compound is hygroscopic and could not be obtained in analytically pure form. The compound was found pure enough for the next step. IR (KBr): $\nu / \mathrm{cm}^{-1}=3405 \mathrm{vs}, 3072$ s, 2933 vs, 2360 m, 2343 m, 1572 vs, 1479 vs, 1384 m, 1309 s, 1147 w, 1090 w, 1038 w, 997 w, 920 m, 859 m, 679 s. ${ }^{1} \mathrm{H}-\mathrm{NMR}$ (400 MHz, $\left.\mathrm{CD}_{3} \mathrm{OD}\right): \delta=2.54\left(\mathrm{t},{ }^{3} J=6.0 \mathrm{~Hz}, 4 \mathrm{H},\left(\mathrm{H}_{2} \mathrm{NCH}_{2}\right)\right.$, $2.69\left(\mathrm{t},{ }^{3} \mathrm{~J}=6.0 \mathrm{~Hz}, 4 \mathrm{H},\left(\mathrm{CH}_{2}\right)_{2} \mathrm{~N}\right), 3.14\left(\mathrm{~d},{ }^{3} \mathrm{~J}=6.4 \mathrm{~Hz}, 2 \mathrm{H}\right.$, $\left.\mathrm{NCH}_{2} \mathrm{CH}=\mathrm{CH}_{2}\right), 5.17\left(\mathrm{~m}, 1 \mathrm{H}, \mathrm{CH}=\mathrm{CH}_{2}\right), 5.89\left(\mathrm{~m}, 2 \mathrm{H}, \mathrm{CH}=\mathrm{CH}_{2}\right)$. ${ }^{13} \mathrm{C}\left\{{ }^{1} \mathrm{H}\right\}-\mathrm{NMR}\left(100 \mathrm{MHz}, \mathrm{CD}_{3} \mathrm{OD}\right): \delta=38.57\left(\mathrm{H}_{2} \mathrm{NCH}_{2}\right), 55.96$ $\left(\left(\mathrm{CH}_{2}\right)_{2} \mathrm{~N}\right), 57.12\left(\mathrm{NCH}_{2}\right), 116.70\left(\mathrm{CH}=\mathrm{CH}_{2}\right), 135.27\left(\mathrm{CH}=\mathrm{CH}_{2}\right)$.

Bisallylated aza-thioether 14. A solution of $\mathrm{N}$-allyl-bis(2aminoethyl)-amine 13 (702 mg, $4.61 \mathrm{mmol})$ in EtOH $(150 \mathrm{~mL}$, $3: 1, \mathrm{v}: \mathrm{v})$ and a solution of 1,2-bis(4-tert-butyl-2,6-diformylphenylthio)ethane $10(1.08 \mathrm{~g}, 2.30 \mathrm{mmol})$ in $\mathrm{CH}_{2} \mathrm{Cl}_{2}(500 \mathrm{~mL})$ were added simultaneously over the course of $3 \mathrm{~h}$ into a EtOH/ $\mathrm{CH}_{2} \mathrm{Cl}_{2}(600 \mathrm{~mL}, 1: 3 \mathrm{v}: \mathrm{v})$ solvent mixture. After the resulting mixture was stirred for further $2 \mathrm{~d}$, the $\mathrm{CH}_{2} \mathrm{Cl}_{2}$ solvent was removed under reduced pressure. Sodium borohydride $(690 \mathrm{mg}$, $18.24 \mathrm{mmol}$ ) was added and the mixture was stirred at r.t. for another $2 \mathrm{~h}$. The excess reducing agent was destroyed by adding $\mathrm{HCl}$ conc (final $\mathrm{pH}=1$ ). The mixture was subsequently evaporated to dryness, re-dissolved in $\mathrm{CH}_{2} \mathrm{Cl}_{2} / \mathrm{H}_{2} \mathrm{O}(100 \mathrm{~mL}$, $1: 1 \mathrm{v}: \mathrm{v})$, and the $\mathrm{pH}$ was adjusted to $\sim 13$ with aqueous $\mathrm{KOH}$ (5 M). After stirring for $2 \mathrm{~h}$, the layers were separated and the aqueous phase was extracted with dichloromethane $(4 \times$ $150 \mathrm{~mL}$ ). The organic fractions were combined and dried with anhydrous $\mathrm{K}_{2} \mathrm{CO}_{3}$. Evaporation of the solvent gave a foam, which was recrystallized from EtOH to give $1.41 \mathrm{~g}$ (88\%) of the title compound. Found: C 68.90, H 9.29, N 12.29, S 9.11; $\mathrm{C}_{40} \mathrm{H}_{64} \mathrm{~N}_{6} \mathrm{~S}_{2}$ (693.11) requires: C 69.32, H 9.31, N 12.13, S 9.25. IR (KBr): $\nu / \mathrm{cm}^{-1}=3424 \mathrm{~m}, 3261 \mathrm{~m}, 2955 \mathrm{vs}, 2902 \mathrm{~s}, 2867 \mathrm{~s}, 2803$ vs, $1599 \mathrm{~m}, 1577 \mathrm{~m}, 1451 \mathrm{vs}, 1417 \mathrm{~s}, 1366 \mathrm{~s}, 1330 \mathrm{w}, 1293 \mathrm{w}$, 1262 m, 1230 w, 1205 m, 1149 s, 1094 s, 1054 s, 1029 s, 921 m, 897 w, 804 s. ${ }^{1} \mathrm{H}-\mathrm{NMR}\left(300 \mathrm{MHz}, \mathrm{CDCl}_{3}\right): \delta=1.30(\mathrm{~s}, 18 \mathrm{H}$, $\left.\mathrm{C}\left(\mathrm{CH}_{3}\right)_{3}\right), 2.81\left(\mathrm{~s}, 4 \mathrm{H}, \mathrm{SCH}_{2}\right), 2.96\left(\mathrm{~m}, 8 \mathrm{H},\left(\mathrm{NCH}_{2} \mathrm{CH}_{2}\right)_{2} \mathrm{~N}\right), 3.14$ $\left(\mathrm{m}, 8 \mathrm{H},\left(\mathrm{NCH}_{2} \mathrm{CH}_{2}\right)_{2} \mathrm{~N}\right), 3.30\left(\mathrm{~d}, J=6.6 \mathrm{~Hz}, 4 \mathrm{H}, \mathrm{CH}_{2} \mathrm{CH}=\mathrm{CH}_{2}\right)$, $4.12\left(\mathrm{~s}, 8 \mathrm{H}, \mathrm{ArCH}_{2}\right), 5.21(\mathrm{~m}, 2 \mathrm{H}, \mathrm{CH}=\mathrm{CHH}), 5.23(\mathrm{~m}, 2 \mathrm{H}$, $\mathrm{CH}=\mathrm{CH} H), 5.90\left(\mathrm{~m}, 2 \mathrm{H}, \mathrm{CH}=\mathrm{CH}_{2}\right), 6.32(\mathrm{~s}(\mathrm{br}), 4 \mathrm{H}, \mathrm{NH})$, $7.72(\mathrm{~s}, 4 \mathrm{H}, \mathrm{Ar} H) .{ }^{13} \mathrm{C}\left\{{ }^{1} \mathrm{H}\right\}-\mathrm{NMR}\left(75 \mathrm{MHz}, \mathrm{CDCl}_{3}\right): \delta=31.36$ $\left(\mathrm{C}\left(\mathrm{CH}_{3}\right)_{3}\right), 35.20\left(\mathrm{C}\left(\mathrm{CH}_{3}\right)_{3}\right), 37.58\left(\mathrm{SCH}_{2}\right)_{2}, 49.02\left(\left(\mathrm{NCH}_{2} \mathrm{CH}_{2}\right)_{2} \mathrm{~N}\right)$, $50.31\left(\left(\mathrm{NCH}_{2} \mathrm{CH}_{2}\right)_{2} \mathrm{~N}\right), 51.41\left(\mathrm{ArCH}_{2}\right), 60.20\left(\mathrm{NCH}_{2} \mathrm{CH}=\mathrm{CH}_{2}\right)$, $119.32\left(\mathrm{CH}=\mathrm{CH}_{2}\right), 128.34\left(\mathrm{ArC}-3,3^{\prime}\right), 130.07\left(\mathrm{ArC}-2,2^{\prime}\right), 134.56$ $\left(\mathrm{CH}=\mathrm{CH}_{2}\right), 140.16$ (ArC-1), $154.18(\mathrm{ArC}-4)$.

Hexa-(cyanoethylated) azathioether 15. The thioether 1 (4.67 g, $7.62 \mathrm{mmol}$ ) was dissolved in acrylonitrile $(3 \mathrm{~mL})$ and the resulting mixture was stirred for $3 \mathrm{~d}$ at $80{ }^{\circ} \mathrm{C}$. The excess acrylonitrile was evaporated, and the yellow solid was dissolved in $100 \mathrm{~mL}$ of a $2: 1 \mathrm{CH}_{2} \mathrm{Cl}_{2} / \mathrm{CH}_{3} \mathrm{CN}$ solvent mixture. Evaporation of the $\mathrm{CH}_{2} \mathrm{Cl}_{2}$ provided a colorless solid, which was filtered and dried in air.
Yield: $7.08 \mathrm{~g}$ (7.6 mmol, 99\%), colorless solid. M.p. $170-172{ }^{\circ} \mathrm{C}$. Found: C 66.83, H 7.92, N 17.82; $\mathrm{C}_{52} \mathrm{H}_{74} \mathrm{~N}_{12} \mathrm{~S}_{2}$ (931.37) requires: C 67.06, $\mathrm{H}$ 8.01, $\mathrm{N}$ 18.05. $\mathrm{m} / \mathrm{z}$ (ESI+, MeOH): $\mathrm{C}_{52} \mathrm{H}_{74} \mathrm{~N}_{12} \mathrm{~S}_{2}$ (930.56) $\left[\mathrm{M}+\mathrm{H}^{+}\right]^{+}$calcd: 931.57; found 931.56. IR (KBr): $\nu / \mathrm{cm}^{-1}=3426 \mathrm{vw}, 3054 \mathrm{~m}, 2951 \mathrm{vs}, 2818 \mathrm{vs}, 2246 \mathrm{vs} \nu(\mathrm{CN})$, $1593 \mathrm{~m}, 1556 \mathrm{vw}, 1477 \mathrm{~m}, 1464 \mathrm{~s}, 1434 \mathrm{~s}, 1424 \mathrm{~s}, 1405 \mathrm{~m}$, $1381 \mathrm{~m}, 1356 \mathrm{~s}, 1328 \mathrm{~m}, 1292 \mathrm{~m}, 1276 \mathrm{~s}, 1248 \mathrm{~m}, 1215 \mathrm{~m}, 1171 \mathrm{w}$, 1139 s, 1110 vs, 1047 s, 1005 m, 987 m, 961 m, 947 m, 887 s, $832 \mathrm{w}, 801 \mathrm{vw}, 774 \mathrm{~m}, 733 \mathrm{~m}, 704 \mathrm{vw}, 681 \mathrm{vw}, 650 \mathrm{w}, 596 \mathrm{w}$. ${ }^{1} \mathrm{H}-\mathrm{NMR}\left(300 \mathrm{MHz}, \mathrm{CDCl}_{3}\right): \delta=1.33\left(\mathrm{~s}, 18 \mathrm{H}, \operatorname{ArC}\left(\mathrm{CH}_{3}\right)_{3}\right), 2.38$ $\left(\mathrm{m}, 8 \mathrm{H}, \mathrm{CH}_{2} \mathrm{CN}\right), 2.40\left(\mathrm{~m}, 4 \mathrm{H}, \mathrm{CH}_{2} \mathrm{CN}\right), 2.66(\mathrm{~m}, 8 \mathrm{H}$, $\left.\left(\mathrm{NCH}_{2} \mathrm{CH}_{2}\right)_{2} \mathrm{~N}\right), 2.67\left(\mathrm{~s}, 4 \mathrm{H}, \mathrm{SCH}_{2}\right), 2.68\left(\mathrm{~m}, 8 \mathrm{H},\left(\mathrm{NCH}_{2} \mathrm{CH}_{2}\right)_{2} \mathrm{~N}\right)$, $2.73\left(\mathrm{~m}, 8 \mathrm{H}, \mathrm{CH}_{2} \mathrm{CH}_{2} \mathrm{CN}\right), 2.78\left(\mathrm{~m}, 4 \mathrm{H}, \mathrm{CH}_{2} \mathrm{CH}_{2} \mathrm{CN}\right), 3.76$ (s, $\left.8 \mathrm{H}, \mathrm{ArCH}_{2}\right), 7.53(\mathrm{~s}, 4 \mathrm{H}, \mathrm{ArH}) .{ }^{13} \mathrm{C}\left\{{ }^{1} \mathrm{H}\right\}-\mathrm{NMR}(75 \mathrm{MHz}$, $\left.\mathrm{CDCl}_{3}\right): \delta=17.03\left(\mathrm{CH}_{2} \mathrm{CN}\right), 17.38\left(\mathrm{CH}_{2} \mathrm{CN}\right), 31.46\left(\mathrm{C}\left(\mathrm{CH}_{3}\right)_{3}\right)$, $35.00\left(\mathrm{C}\left(\mathrm{CH}_{3}\right)_{3}\right), \quad 36.16\left(\mathrm{SCH}_{2}\right), 47.3 \quad\left(\mathrm{ArCH}_{2} \mathrm{NCH}_{2} \mathrm{CH}_{2} \mathrm{CN}\right)$, $51.82\left(\mathrm{CH}_{2} \mathrm{CH}_{2} \mathrm{CN}\right), 52.39\left(\left(\mathrm{NCH}_{2} \mathrm{CH}_{2}\right)_{2} \mathrm{~N}\right), 52.96\left(\left(\mathrm{NCH}_{2} \mathrm{CH}_{2}\right)_{2} \mathrm{~N}\right)$, $63.1\left(\mathrm{ArCH}_{2} \mathrm{~N}\right), 118.90\left(\mathrm{ArCH}_{2} \mathrm{NCH}_{2} \mathrm{CH}_{2} \mathrm{CN}\right), 118.96(\mathrm{CN})$, $125.20\left(\mathrm{ArC}-3,3^{\prime}\right), 128.17\left(\mathrm{ArC}-2,2^{\prime}\right), 142.71$ (ArC-1), 152.26 $(\mathrm{ArC}-4)$. This compound was additionally characterized by X-ray crystallography.

Hexa(3-aminopropylated) aza-thioether 16. A suspension of $\mathrm{LiBH}_{4}$ (0.48 g, $\left.22 \mathrm{mmol}\right), \mathrm{Me}_{3} \mathrm{SiCl}(4.45 \mathrm{~g}, 41 \mathrm{mmol})$, and the nitrile $15(0.50 \mathrm{~g}, 0.54 \mathrm{mmol})$ in $300 \mathrm{~mL}$ of dry THF was stirred for $10 \mathrm{~h}$ at $50{ }^{\circ} \mathrm{C}$. The mixture was refluxed for further $12 \mathrm{~h}$, cooled to r.t., and quenched with $\mathrm{MeOH}$ to give a clear solution. The solution was stirred for $1 \mathrm{~h}$, evaporated to dryness, and suspended in $40 \mathrm{~mL}$ of $3 \mathrm{M} \mathrm{NaOH}$ solution. The aqueous phase was extracted with $\mathrm{CH}_{2} \mathrm{Cl}_{2}(4 \times 20 \mathrm{~mL})$. The organic fractions were combined and dried with anhydrous $\mathrm{K}_{2} \mathrm{CO}_{3}$. Evaporation of the solvent gave $\mathbf{1 6}$ as a colorless solid (376 mg, 73\%). The compound is hygroscopic and could not be obtained in analytically pure form, but the spectroscopic data (see $\mathrm{ESI} \dagger$ ) prove the formulation of this compound. $m / z$ (ESI+, $\mathrm{MeOH}$ ): $\mathrm{C}_{52} \mathrm{H}_{98} \mathrm{~N}_{12} \mathrm{~S}_{2}$ (955.56) $\left[\mathrm{M}+\mathrm{H}^{+}\right]^{+}$calcd: 955.76; found 955.76. IR (KBr): $\nu / \mathrm{cm}^{-1}=$ 3360 (w, NH NH, 3051 m, 2963 s, 1593 (w, NH $), 1261$ s, 1097 (s, C-S), 1021 s, 799 s. ${ }^{1} \mathrm{H}-\mathrm{NMR}\left(700 \mathrm{MHz}, \mathrm{CDCl}_{3}\right): \delta=1.26(\mathrm{~s}, 18 \mathrm{H}$, $\left.\mathrm{C}\left(\mathrm{CH}_{3}\right)_{3}\right), 1.49\left(\mathrm{tt},{ }^{3} J=6.7,{ }^{3} J=6.6 \mathrm{~Hz}, 8 \mathrm{H}, \mathrm{CH}_{2} \mathrm{CH}_{2} \mathrm{CH}_{2} \mathrm{NH}_{2}\right), 1.56$ $\left(\mathrm{tt},{ }^{3} J=7.0,{ }^{3} J=6.7 \mathrm{~Hz}, 4 \mathrm{H}, \mathrm{CH}_{2} \mathrm{CH}_{2} \mathrm{CH}_{2} \mathrm{NH}_{2}\right), 1.64\left(\mathrm{~s} \mathrm{br}, 6 \mathrm{H}, \mathrm{NH}_{2}\right.$ ), $2.35\left(\mathrm{t},{ }^{3} \mathrm{~J}=6.6 \mathrm{~Hz}, 8 \mathrm{H}, \mathrm{CH}_{2} \mathrm{CH}_{2} \mathrm{CH}_{2} \mathrm{NH}_{2}\right), 2.47\left(\mathrm{t},{ }^{3} \mathrm{~J}=7.0 \mathrm{~Hz}, 4 \mathrm{H}\right.$, $\mathrm{CH}_{2} \mathrm{CH}_{2} \mathrm{CH}_{2} \mathrm{NH}_{2}$ ), $2.53\left(\mathrm{~m}, 16 \mathrm{H}, \mathrm{NCH}_{2} \mathrm{CH}_{2} \mathrm{~N}\right.$ ), 2.55 (s, $4 \mathrm{H}, \mathrm{SCH}_{2}$ ), 3.51 (s, $\left.8 \mathrm{H}, \mathrm{ArCH}_{2}\right), 7.41$ (s, $\left.4 \mathrm{H}, \mathrm{ArH}\right) .{ }^{13} \mathrm{C}\left\{{ }^{1} \mathrm{H}\right\}-\mathrm{NMR}(100 \mathrm{MHz}$, $\left.\mathrm{CDCl}_{3}\right): \delta=30.25\left(\mathrm{ArCH}_{2} \mathrm{NCH}_{2} \mathrm{CH}_{2} \mathrm{CH}_{2} \mathrm{NH}_{2}\right), 30.42\left(\mathrm{C}\left(\mathrm{CH}_{3}\right)_{3}\right), 30.56$ $\left(\mathrm{CH}_{2} \mathrm{CH}_{2} \mathrm{NH}_{2}\right), 33.77\left(C\left(\mathrm{CH}_{3}\right)_{3}\right), 34.32\left(\mathrm{SCH}_{2}\right), 39.48\left(\mathrm{CH}_{2} \mathrm{NH}_{2}\right)$, $39.48\left(\mathrm{CH}_{2} \mathrm{NH}_{2}\right), 50.25\left(\mathrm{ArCH}_{2} \mathrm{NCH}_{2} \mathrm{CH}_{2} \mathrm{CH}_{2} \mathrm{~N}\right), 53.37\left(\mathrm{NCH}_{2} \mathrm{CH}_{2}-\right.$ $\left.\mathrm{CH}_{2} \mathrm{NH}_{2}\right), 56.65\left(\mathrm{ArCH}_{2}\right), 123.25\left(\mathrm{ArC}-3,3^{\prime}\right), 126.60\left(\mathrm{ArC}-2,2^{\prime}\right)$, 142.45 (ArC-1), 150.12 (ArC-4).

Hexa(2-methoxyacetylated) aza-thioether 17. Compound 1 $(2.50 \mathrm{~g}, 4.08 \mathrm{mmol})$ in dry $\mathrm{CHCl}_{3}(20 \mathrm{~mL}), 2$-methoxyacetyl chloride (2.86 g, $26.3 \mathrm{mmol})$, and triethylamine (2.48 g, $24.5 \mathrm{mmol})$ were reacted to give a colorless solution, which was stirred for $12 \mathrm{~h}$ and evaporated to dryness. The residue was suspended in THF $(10 \mathrm{~mL})$, filtered, and dried. The colorless solid was purified by recrystallization from EtOH. Yield: $3.38 \mathrm{~g}$ (79\%). Found: C 59.40, $\mathrm{H} 7.35, \mathrm{~N} 7.89, \mathrm{~S} 5.88 ; \mathrm{C}_{52} \mathrm{H}_{80} \mathrm{~N}_{6} \mathrm{O}_{12} \mathrm{~S}_{2}(1045.36)$ requires: $\mathrm{C}$ 59.75, $\mathrm{H}$ 7.71, $\mathrm{N}$ 8.04, $\mathrm{S}$ 6.13. $\mathrm{m} / \mathrm{z}$ (ESI+, $\mathrm{MeOH})$ : 
$\mathrm{C}_{52} \mathrm{H}_{80} \mathrm{~N}_{6} \mathrm{O}_{12} \mathrm{~S}_{2}$ (1044.53) $\left[\mathrm{M}+\mathrm{H}^{+}\right]^{+}$calcd: 1045.54; found 1045.5. IR (KBr): $\nu / \mathrm{cm}^{-1}=3483 \mathrm{~m}, 2954 \mathrm{~s}, 2824 \mathrm{~m}, 1655$ [vs, $\nu(\mathrm{CO})$ ], 1560 w, 1468 s, 1433 s, 1364 m, 1301 m, 1196 s, 1125 s, 1050 w, $996 \mathrm{w}, 966 \mathrm{w}, 934 \mathrm{w}, 830 \mathrm{vw}, 801 \mathrm{vw}, 773 \mathrm{vw} 729 \mathrm{vw}, 686 \mathrm{vw}$. ${ }^{1} \mathrm{H}-\mathrm{NMR}\left(600 \mathrm{MHz}, 400 \mathrm{~K}, \mathrm{DMSO}-\mathrm{d}_{6}\right): \delta=1.20\left(\mathrm{~s}, 18 \mathrm{H}, \mathrm{C}\left(\mathrm{CH}_{3}\right)\right.$ ), $3.01\left(\mathrm{~s}, 4 \mathrm{H}, \mathrm{SCH}_{2}\right), 3.34\left(\mathrm{~s}, 12 \mathrm{H}, \mathrm{OCH}_{3}\right), 3.40\left(\mathrm{~s}, 6 \mathrm{H}, \mathrm{OCH}_{3}\right), 3.46$ $\left(\mathrm{s}, 16 \mathrm{H}, \mathrm{NCH}_{2} \mathrm{CH}_{2} \mathrm{~N}\right), 4.10\left(\mathrm{~s}, 8 \mathrm{H}, \mathrm{CH}_{2} \mathrm{O}\right), 4.18(\mathrm{~s}, 4 \mathrm{H}$, $\mathrm{CH}_{2} \mathrm{OCH}_{3}$ ), 4.74 (s, $8 \mathrm{H}, \mathrm{ArCH}_{2}$ ), 6.99 (s, $\left.4 \mathrm{H}, \mathrm{Ar}-\mathrm{H}\right) .{ }^{13} \mathrm{C}\left\{{ }^{1} \mathrm{H}\right\}-$ NMR (150 MHz, $\left.400 \mathrm{~K}, \mathrm{DMSO}-\mathrm{d}_{6}\right): \delta=29.81\left(\mathrm{C}\left(\mathrm{CH}_{3}\right)_{3}\right), 33.56$ $\left(\mathrm{C}\left(\mathrm{CH}_{3}\right)_{3}\right), 35.29\left(\mathrm{SCH}_{2}\right), 44.89\left(\mathrm{br}, \mathrm{NCH}_{2} \mathrm{CH}_{2} \mathrm{~N}\right), 48.49\left(\mathrm{ArCH}_{2}\right)$, $57.68\left(\mathrm{OCH}_{3}\right), 57.73\left(\mathrm{OCH}_{3}\right), 70.12\left(\mathrm{OCH}_{2} \mathrm{O}\right), 70.21\left(\mathrm{OCH}_{2} \mathrm{O}\right)$, $121.17\left(\mathrm{ArC}-3,3^{\prime}\right), 125.82\left(\mathrm{ArC}-2,2^{\prime}\right), 140.70$ (ArC-1), 151.66 (ArC-4), 168.62 (CO), 168.69 (CO). This compound was additionally characterized by X-ray crystallography.

Hexa(2-methoxyethylated) aza-thioether 18. Compound 17 $(3.03 \mathrm{~g}, 2.90 \mathrm{mmol})$ in THF $(20 \mathrm{~mL}), \mathrm{LiBH}_{4}(827 \mathrm{mg}, 38.0 \mathrm{mmol})$ and $\mathrm{Me}_{3} \mathrm{SiCl}(8.10 \mathrm{~g}, 74.6 \mathrm{mmol})$ in THF $(20 \mathrm{~mL})$ were reacted under $\mathrm{N}_{2}$ to give a colorless solution which was stirred for $12 \mathrm{~h}$, quenched with $\mathrm{MeOH}(2 \mathrm{~mL})$ and evaporated to dryness. The residue was triturated with aqueous lithium hydroxide solution ( $3 \mathrm{M}, 20 \mathrm{~mL}$ ) and $\mathrm{CH}_{2} \mathrm{Cl}_{2}(50 \mathrm{~mL})$, the layers were separated and the aqueous phase was extracted with $\mathrm{CH}_{2} \mathrm{Cl}_{2}(3 \times 10 \mathrm{~mL})$. The organic fractions were combined and dried with anhydrous $\mathrm{MgSO}_{4}$. Evaporation of the solvent gave $\mathbf{1 8}$ as a white solid (2.03 g, 73\%). Slow evaporation of a $\mathrm{CH}_{2} \mathrm{Cl}_{2} / \mathrm{MeCN}(1: 1)$ solution afforded colorless crystals. Found: C 64.76, H 9.89, $\mathrm{N}$ 8.65, S 6.50; $\mathrm{C}_{52} \mathrm{H}_{92} \mathrm{~N}_{6} \mathrm{O}_{6} \mathrm{~S}_{2}$ (961.46) requires: C 64.96, $\mathrm{H}$ 9.65, $\mathrm{N}$ 8.74, S 6.67. $m / z$ (ESI+, MeOH): $\mathrm{C}_{52} \mathrm{H}_{92} \mathrm{~N}_{6} \mathrm{O}_{6} \mathrm{~S}_{2}$ (960.55) $[\mathrm{M}+$ $\left.\mathrm{H}^{+}\right]^{+}$calcd: 961.66; found 961.7. IR $(\mathrm{KBr}): \nu / \mathrm{cm}^{-1}=3425 \mathrm{~s}, 2953$ vs, 2871 vs, 2816 vs, 2336 vw, 1954 vw, 1667 m, 1594 m,1559 w, 1451 vs, 1404 s, 1362 vs, 1297 s, 1261 m, 1199 s, 1119 vs, 1080 vs, $1018 \mathrm{~s}, 958 \mathrm{~m}, 927 \mathrm{~m}, 894 \mathrm{~m}, 781 \mathrm{w}, 733 \mathrm{w}, 704 \mathrm{w}, 647 \mathrm{w}$, 558 w. ${ }^{1} \mathrm{H}-\mathrm{NMR}\left(600 \mathrm{MHz}, 400 \mathrm{~K}, \mathrm{DMSO}^{-\mathrm{d}_{6}}\right): \delta=1.32(\mathrm{~s}, 18 \mathrm{H}$, $\left.\mathrm{C}\left(\mathrm{CH}_{3}\right)\right), 2.64\left(\mathrm{t},{ }^{3} \mathrm{~J}=5.3,8 \mathrm{H}, \mathrm{NCH}_{2} \mathrm{CH}_{2} \mathrm{OCH}_{3}\right), 2.73(\mathrm{~m}, 4 \mathrm{H}$, $\left.\left.\mathrm{NCH}_{2} \mathrm{CH}_{2} \mathrm{OCH}_{3}\right)\right), 2.75\left(\mathrm{~s}, 4 \mathrm{H}, \mathrm{SCH}_{2}\right), 2.85\left(\mathrm{~m}, 16 \mathrm{H}, \mathrm{NCH}_{2}-\right.$ $\left.\mathrm{CH}_{2} \mathrm{~N}\right), 3.28\left(\mathrm{~s}, 12 \mathrm{H}, \mathrm{OCH}_{3}\right), 3.31\left(\mathrm{~s}, 6 \mathrm{H}, \mathrm{OCH}_{3}\right), 3.41\left(\mathrm{t},{ }^{3} \mathrm{~J}=5.3\right.$, $\left.8 \mathrm{H}, \mathrm{CH}_{2} \mathrm{OCH}_{3}\right), 3.60\left(\mathrm{~m}, 4 \mathrm{H}, \mathrm{CH}_{2} \mathrm{OCH}_{3}\right), 3.78\left(\mathrm{~s}, 8 \mathrm{H}, \mathrm{ArCH}_{2}\right)$, 7.53 (s, $4 \mathrm{H}, \mathrm{ArH}) .{ }^{13} \mathrm{C}\left\{{ }^{1} \mathrm{H}\right\}-\mathrm{NMR}\left(100 \mathrm{MHz}, \mathrm{CDCl}_{3}\right): \delta=31.32$ $\left(\mathrm{C}\left(\mathrm{CH}_{3}\right)_{3}\right), 34.68\left(\mathrm{C}\left(\mathrm{CH}_{3}\right)_{3}\right), 35.57\left(\mathrm{SCH}_{2}\right), 50.82\left(\mathrm{NCH}_{2} \mathrm{CH}_{2} \mathrm{OCH}_{3}\right)$, $50.92\left(\left(\mathrm{NCH}_{2} \mathrm{CH}_{2}\right)_{2} \mathrm{~N}\right), \quad 52.74 \quad\left(\mathrm{ArCH}_{2} \mathrm{NCH}_{2} \mathrm{CH}_{2} \mathrm{OCH}_{3}\right), \quad 54.68$ $\left(\left(\mathrm{NCH}_{2} \mathrm{CH}_{2}\right)_{2} \mathrm{~N}\right), 58.17\left(\mathrm{ArCH}_{2} \mathrm{~N}\right), 58.92\left(\mathrm{OCH}_{3}\right), 58.97\left(\mathrm{OCH}_{3}\right)$, $70.50\left(\mathrm{CH}_{2} \mathrm{OCH}_{3}\right), 70.82\left(\mathrm{CH}_{2} \mathrm{OCH}_{3}\right), 125.72\left(\mathrm{ArC}-3,3^{\prime}\right), 128.55$ $\left(\mathrm{ArC}-2,2^{\prime}\right), 142.30$ (ArC-1), 151.87 (ArC-4).

Tetra(2-methoxyacetylated) aza-thioether 19. By analogy to the preparation of 17 , compound $2(1.60 \mathrm{~g}, 2.50 \mathrm{mmol})$ in dry chloroform (20 mL), 2-methoxyacetyl chloride (1.14 g, $10.5 \mathrm{mmol})$, and triethylamine $(1.10 \mathrm{~g}, 10.0 \mathrm{mmol})$ were reacted to give a colorless solution, which was stirred for $12 \mathrm{~h}$ and evaporated to dryness. The residue was suspended in THF $(10 \mathrm{~mL})$, filtered, and dried. The colorless solid was purified by recrystallization from EtOH. Yield: $2.00 \mathrm{~g}$ (86\%). Found: C 61.80, H 8.25, N 8.89, S 6.65; $\mathrm{C}_{48} \mathrm{H}_{76} \mathrm{~N}_{6} \mathrm{O}_{8} \mathrm{~S}_{2}$ (929.29) requires: $\mathrm{C}$ 62.04, $\mathrm{H}$ 8.24, N 9.04, S 6.90. $m / z$ (ESI+, MeOH): $\mathrm{C}_{48} \mathrm{H}_{76} \mathrm{~N}_{6} \mathrm{O}_{8} \mathrm{~S}_{2}$ (928.52) $\left[\mathrm{M}+\mathrm{H}^{+}\right]^{+}$calcd: 929.52; found 929.52. IR (KBr): $\nu / \mathrm{cm}^{-1}=3449 \mathrm{~s}, 2958 \mathrm{vs}, 2822 \mathrm{~m}$, 1655 vs $\nu$ CO, 1469 vs, 1430 vs, 1361 m, 1296 m, 1197 s, 1125 vs, 1068 m, 1019 m, 972 w, 937 m, 800 w, 750 w, 726 w, 687 w, 596 m.
${ }^{1} \mathrm{H}-\mathrm{NMR}\left(600 \mathrm{MHz}, 400 \mathrm{~K}, \mathrm{DMSO}-\mathrm{d}_{6}\right): \delta=1.21\left(\mathrm{~s}, 18 \mathrm{H}, \mathrm{C}\left(\mathrm{CH}_{3}\right)_{3}\right)$, $2.43\left(\mathrm{~s}, 6 \mathrm{H}, \mathrm{NCH}_{3}\right), 2.76\left(\mathrm{~m}, 8 \mathrm{H},\left(\mathrm{NCH}_{2} \mathrm{CH}_{2}\right)_{2} \mathrm{NCH}_{3}\right), 3.03(\mathrm{~s}, 4 \mathrm{H}$, $\left.\mathrm{SCH}_{2}\right), 3.32\left(\mathrm{~s}, 12 \mathrm{H}, \mathrm{OCH}_{3}\right), 3.45\left(\mathrm{~s}, 8 \mathrm{H},\left(\mathrm{NCH}_{2} \mathrm{CH}_{2}\right)_{2} \mathrm{NCH}_{3}\right), 4.80(\mathrm{~s}$, $8 \mathrm{H}, \mathrm{ArCH}_{2}$ ), 7.05 (s, $\left.4 \mathrm{H}, \mathrm{ArH}\right) .{ }^{13} \mathrm{C}\left\{{ }^{1} \mathrm{H}\right\}-\mathrm{NMR}(150 \mathrm{MHz}, 400 \mathrm{~K}$,

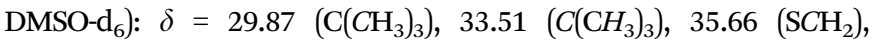
$\left.41.91\left(\mathrm{NCH}_{3}\right), 44.35\left(\mathrm{NCH}_{2} \mathrm{CH}_{2} \mathrm{~N}\right), 48.53 \quad\left(\mathrm{NCH}_{2} \mathrm{CH}_{2}\right)_{2} \mathrm{NCH}_{3}\right)$, $54.85\left(\mathrm{ArCH}_{2}\right), \quad 57.64\left(\mathrm{OCH}_{3}\right), 70.35\left(\mathrm{NCOCH}_{2} \mathrm{OCH}_{3}\right), 121.59$ (ArC-3,3'), 126.21 (ArC-2,2'), 140.79 (ArC-1), 151.44 (ArC-4), 168.25 (CO). This compound was additionally characterized by X-ray crystallography.

Tetra(2-methoxyethylated) aza-thioether 20. By analogy to the preparation of $\mathbf{1 8}$, amide $19(1.28 \mathrm{~g}, 1.38 \mathrm{mmol})$ in THF $(20 \mathrm{~mL}), \mathrm{LiBH}_{4}(152 \mathrm{mg}, 6.98 \mathrm{mmol})$ and $\mathrm{Me}_{3} \mathrm{SiCl}(1.49 \mathrm{~g}$, $13.7 \mathrm{mmol})$ in THF $(20 \mathrm{~mL})$ were reacted under $\mathrm{N}_{2}$ to give a colorless solution which was stirred for $12 \mathrm{~h}$, quenched with $\mathrm{MeOH}$ and evaporated to dryness. The residue was triturated with aqueous $\mathrm{LiOH}(3 \mathrm{M}, 20 \mathrm{~mL})$ and $\mathrm{CH}_{2} \mathrm{Cl}_{2}(50 \mathrm{~mL})$, the layers were separated and the aqueous phase was extracted with $\mathrm{CH}_{2} \mathrm{Cl}_{2}(3 \times 10 \mathrm{~mL})$. The organic fractions were combined and dried with anhydrous $\mathrm{MgSO}_{4}$. Evaporation of the solvent gave 20 as a white solid $(0.88 \mathrm{~g}, 73 \%)$. Slow evaporation of a $\mathrm{CH}_{2} \mathrm{Cl}_{2} / \mathrm{MeCN}(1: 1)$ solution afforded colorless crystals. Found: C 66.13, $\mathrm{H}$ 9.78, $\mathrm{N}$ 9.41, $\mathrm{S}$ 7.22; $\mathrm{C}_{48} \mathrm{H}_{84} \mathrm{~N}_{6} \mathrm{O}_{4} \mathrm{~S}_{2}$ (873.36) requires: C 66.01, H 9.69, N 9.62, S 7.34. $\mathrm{m} / z(\mathrm{ESI}+, \mathrm{MeOH}): \mathrm{C}_{48} \mathrm{H}_{84} \mathrm{~N}_{6} \mathrm{O}_{4} \mathrm{~S}_{2}$ (872.60) $\left[\mathrm{M}+\mathrm{H}^{+}\right]^{+}$calcd: 873.61; found 873.60. IR ( $\left.\mathrm{KBr}\right)$ : $\nu / \mathrm{cm}^{-1}=3425$ s, $2953 \mathrm{vs}, 2871 \mathrm{vs}, 2816 \mathrm{vs}, 2336 \mathrm{vw}, 1954 \mathrm{vw}$, 1667 m, 1594 m, 1559 w, 1451 vs, 1404 s, 1362 vs, 1297 s, $1261 \mathrm{~m}, 1199 \mathrm{~s}, 1119 \mathrm{vs}, 1080 \mathrm{vs}, 1018 \mathrm{~s}, 958 \mathrm{~m}, 927 \mathrm{~m}, 894 \mathrm{~m}$, 781 w, 733 w, 704 w, 647 w, 558 w. ${ }^{1} \mathrm{H}-\mathrm{NMR}(600 \mathrm{MHz}, 400 \mathrm{~K}$, DMSO-d $\left.{ }_{6}\right): \delta=1.30\left(\mathrm{~s}, 18 \mathrm{H}, \mathrm{C}\left(\mathrm{CH}_{3}\right)_{3}\right), 2.25\left(\mathrm{~s}, 6 \mathrm{H}, \mathrm{NCH}_{3}\right)$, $2.50\left(\mathrm{~s}(\mathrm{br}), 8 \mathrm{H},\left(\mathrm{NCH}_{2} \mathrm{CH}_{2}\right)_{2} \mathrm{NCH}_{3}\right), 2.60\left(\mathrm{t},{ }^{3} \mathrm{~J}=9.8,8 \mathrm{H}\right.$, $\mathrm{NCH}_{2} \mathrm{CH}_{2} \mathrm{OCH}_{3}$ ), 2.68 (s (br), $\left.8 \mathrm{H},\left(\mathrm{NCH}_{2} \mathrm{CH}_{2}\right)_{2} \mathrm{NCH}_{3}\right), 2.79$ (s, $\left.4 \mathrm{H}, \mathrm{SCH}_{2}\right), 3.28\left(\mathrm{~s}, 12 \mathrm{H}, \mathrm{OCH}_{3}\right), 3.39\left(\mathrm{t},{ }^{3} \mathrm{~J}=9.8,8 \mathrm{H}, \mathrm{NCH}_{2}-\right.$ $\mathrm{CH}_{2} \mathrm{OCH}_{3}$ ), $3.80\left(\mathrm{~s}, 8 \mathrm{H}, \mathrm{ArCH}_{2}\right), 7.47$ (s, $\left.4 \mathrm{H}, \mathrm{ArH}\right) .{ }^{13} \mathrm{C}\left\{{ }^{1} \mathrm{H}\right\}-\mathrm{NMR}$ $\left(100 \mathrm{MHz}, \mathrm{CDCl}_{3}\right): \delta=31.43\left(\mathrm{C}\left(\mathrm{CH}_{3}\right)_{3}\right), 34.87\left(\mathrm{C}\left(\mathrm{CH}_{3}\right)_{3}\right), 36.16$ $\left(\mathrm{SCH}_{2}\right), 43.42\left(\mathrm{NCH}_{3}\right), 52.35\left(\left(\mathrm{NCH}_{2} \mathrm{CH}_{2}\right)_{2} \mathrm{~N}\right), 53.37\left(\mathrm{ArCH}_{2}-\right.$ $\left.\mathrm{NCH}_{2} \mathrm{CH}_{2} \mathrm{OCH}_{3}\right), \quad 58.57 \quad\left(\mathrm{ArCH}_{2} \mathrm{~N}\right), \quad 58.93 \quad\left(\mathrm{OCH}_{3}\right), \quad 71.50$ $\left(\mathrm{CH}_{2} \mathrm{OCH}_{3}\right)$, (one $\mathrm{CH}_{2}$ signal not observed), $125.70\left(\mathrm{ArC}-3,3^{\prime}\right)$, 128.79 (ArC-2,2'), 143.79 (ArC-1), 151.44 (ArC-4).

Dicarbamoylated macrobicycle 22. To a solution of tert-butyl bis(2-aminoethyl)carbamate $(2.89 \mathrm{~g}, 14.22 \mathrm{mmol})$ in EtOH/ $\mathrm{CHCl}_{3}(800 \mathrm{~mL}, 3: 1 \mathrm{v} / \mathrm{v})$ at $0{ }^{\circ} \mathrm{C}$ was added a solution of 1,2bis(4-tert-butyl-2,6-diformylphenylthio)ethane (3.34 g, $7.11 \mathrm{mmol}$ ) in $\mathrm{CHCl}_{3}(500 \mathrm{~mL})$ over the course of $8 \mathrm{~h}$. After the resulting mixture was stirred for further $2 \mathrm{~d}$, the $\mathrm{CHCl}_{3}$ solvent was removed under reduced pressure. Sodium borohydride $(2.15 \mathrm{~g}, 56.88 \mathrm{mmol})$ was added and the mixture was stirred at r.t. for $18 \mathrm{~h}$. The mixture was evaporated to dryness, then redissolved in $\mathrm{CH}_{2} \mathrm{Cl}_{2} / \mathrm{H}_{2} \mathrm{O}$ $(100 \mathrm{~mL}, 1: 1, \mathrm{v} / \mathrm{v})$, and the $\mathrm{pH}$ was adjusted to $\sim 13$ with aqueous $\mathrm{KOH}(5 \mathrm{M})$. After stirring for $2 \mathrm{~h}$, the layers were separated and the aqueous phase was extracted with $\mathrm{CH}_{2} \mathrm{Cl}_{2}(4 \times 50 \mathrm{~mL})$. The organic fractions were combined and dried with anhydrous $\mathrm{MgSO}_{4}$. Evaporation of the solvent gave an oil, which crystallized from EtOH (10 ml) after standing for 4 weeks. Yield: $1.20 \mathrm{~g}(1.47 \mathrm{mmol}$, 21\%). M.p. $136-138{ }^{\circ}$ C. Found: C 64.75, H 8.58, N 9.75; $\mathrm{C}_{44} \mathrm{H}_{72}$ $\mathrm{N}_{6} \mathrm{O}_{4} \mathrm{~S}_{2}$ 0.5 EtOH $(813.21+23.04)$ requires: C 64.63, H 9.04, 
$\mathrm{N}$ 10.05. $\mathrm{m} / \mathrm{z}(\mathrm{ESI}+): 813.4\left(\mathrm{M}+\mathrm{H}^{+}\right) . \mathrm{IR}(\mathrm{KBr}): \nu / \mathrm{cm}^{-1}=3443 \mathrm{~s}$, $3290 \mathrm{~m} \nu(\mathrm{NH}), 2965 \mathrm{~s}, 2927 \mathrm{~s}, 2865 \mathrm{~m}, 1693 \mathrm{~s} \nu(\mathrm{C}=\mathrm{O}), 1597 \mathrm{w}$, 1478 s, 1458 s, 1411 s, 1365 s, 1249 m, 1228 w, 1173 s, 1154 s, 1091 w, 1043 w, 966 w, 886 w, 858 w, 823 w, 774 w. ${ }^{1} \mathrm{H}-\mathrm{NMR}(400 \mathrm{MHz}$, $\left.\mathrm{CDCl}_{3}\right): \delta=1.29\left(\mathrm{~s}, 18 \mathrm{H}, \operatorname{ArC}\left(\mathrm{CH}_{3}\right)_{3}\right), 1.45\left(\mathrm{~s}, 18 \mathrm{H}, \mathrm{C}\left(\mathrm{CH}_{3}\right)_{3}\right), 2.96$ $\left(\mathrm{t},{ }^{3} \mathrm{~J}=6 \mathrm{~Hz}, 8 \mathrm{H},\left(\mathrm{NCH}_{2} \mathrm{CH}_{2}\right)_{2} \mathrm{~N}\right), 3.20\left(\mathrm{~s}, 4 \mathrm{H}, \mathrm{SCH}_{2}\right), 3.49(\mathrm{t}$, $\left.{ }^{3} J=6 \mathrm{~Hz}, 8 \mathrm{H},\left(\mathrm{NCH}_{2} \mathrm{CH}_{2}\right)_{2} \mathrm{~N}\right), 3.97\left(\mathrm{~s}, 8 \mathrm{H}, \mathrm{ArCH}_{2} \mathrm{~N}\right), 7.28(\mathrm{~s}, 4 \mathrm{H}$, $\mathrm{ArH}) .{ }^{13} \mathrm{C}\left\{{ }^{1} \mathrm{H}\right\}-\mathrm{NMR}\left(100 \mathrm{MHz}, \mathrm{CDCl}_{3}\right): \delta=28.6\left(\mathrm{C}\left(\mathrm{CH}_{3}\right)_{3}\right), 31.4$ $\left(\operatorname{ArC}\left(\mathrm{CH}_{3}\right)_{3}\right), 34.7\left(\operatorname{ArC}\left(\mathrm{CH}_{3}\right)_{3}\right), 37.1\left(\left(\mathrm{ArSCH}_{2}\right)_{2}\right), 47.8\left(\left(\mathrm{NCH}_{2}-\right.\right.$

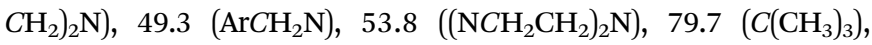
126.4 (ArC-3,3), 130.0 (ArC-2,2), 144.8 (ArC-1), 152.2 (ArC-4), $156.0(C=\mathrm{O})$.

Methylated macrobicycle 23. To a suspension of 22 (1.13 g, $1.39 \mathrm{mmol})$ in $\mathrm{MeOH}(55 \mathrm{~mL})$ was added acetic acid $(4 \mathrm{~mL})$ followed by formaldehyde $(4 \mathrm{ml})$, and sodium cyanoborohydride (689 $\mathrm{mg}, 11.12 \mathrm{mmol}$ ). The resulting clear solution was stirred for $3 \mathrm{~d}$ at r.t., and its $\mathrm{pH}$ was brought to 13 with aqueous $\mathrm{KOH}(5 \mathrm{M})$. The $\mathrm{MeOH}$ was removed under reduced pressure, and $50 \mathrm{~mL} \mathrm{CH}_{2} \mathrm{Cl}_{2} / \mathrm{H}_{2} \mathrm{O}(1: 1 \mathrm{v} / \mathrm{v})$ was added. After stirring for $2 \mathrm{~h}$, the layers were separated and the aqueous phase was extracted with $\mathrm{CH}_{2} \mathrm{Cl}_{2}(4 \times 25 \mathrm{~mL})$. The organic fractions were combined and dried with anhydrous $\mathrm{MgSO}_{4}$. Evaporation gave the crude product, which was purified by recrystallization from $\mathrm{CH}_{2} \mathrm{Cl}_{2} / \mathrm{EtOH}(1: 1 \mathrm{v} / \mathrm{v})$. Yield: $910 \mathrm{mg}$ (1.05 mmol, 75\%), colorless crystals. Mp 217-219 ${ }^{\circ} \mathrm{C}$. Found: C 65.93, $\mathrm{H}$ 9.23, N 9.59; $\mathrm{C}_{48} \mathrm{H}_{80} \mathrm{~N}_{6} \mathrm{O}_{4} \mathrm{~S}_{2}$ (869.32) requires: $\mathrm{C}$ 66.32, $\mathrm{H}$ 9.28, N 9.67. $\mathrm{m} / \mathrm{z}$ (ESI+, $\mathrm{MeOH}): \mathrm{C}_{48} \mathrm{H}_{80} \mathrm{~N}_{6} \mathrm{O}_{4} \mathrm{~S}_{2}$ (868.57) $[\mathrm{M}+$ $\left.\mathrm{H}^{+}\right]^{+}$calcd: 869.58; found 869.50. IR $(\mathrm{KBr}): \nu / \mathrm{cm}^{-1}=3444 \mathrm{~m}$, 2965 s, 2857 m, 2802 s, $2716 \mathrm{w}, 1688 \mathrm{~s}(\nu(\mathrm{C}=\mathrm{O})), 1569 \mathrm{w}$, 1557 w, 1479 s, 1456 s, 1416 s, 1393 m, 1365 s, 1293 m, 1273 m, 1249 m, 1227 m, 1209 m, 1161 s, 1125 m, 1109 m, 1046 m, 1035 m, 1019 m, 984 w, 970 w, 953 w, 912 w, 905 w, 891 m, 864 w, 838 w, 814 w, 804 w, 772 w, 701 w, 685 w, 635 w, 546 w. ${ }^{1} \mathrm{H}-\mathrm{NMR}$ $\left(400 \mathrm{MHz}_{\mathrm{CDCl}}\right): \delta=1.30\left(\mathrm{~s}, 18 \mathrm{H}, \operatorname{ArC}\left(\mathrm{CH}_{3}\right)_{3}\right), 1.48(\mathrm{~s}, 18 \mathrm{H}$, $\left.\mathrm{C}\left(\mathrm{CH}_{3}\right)_{3}\right), 2.08\left(\mathrm{~m}, 12 \mathrm{H}, \mathrm{NCH}_{3}\right), 2.69\left(\mathrm{~m}, 8 \mathrm{H},\left(\mathrm{NCH}_{2} \mathrm{CH}_{2}\right)_{2} \mathrm{~N}\right)$, $3.20\left(\mathrm{~s}, 4 \mathrm{H}, \mathrm{SCH}_{2}\right), 3.40\left(\mathrm{~m}, 8 \mathrm{H},\left(\mathrm{NCH}_{2} \mathrm{CH}_{2}\right)_{2} \mathrm{~N}\right), 3.71(\mathrm{~s}, 8 \mathrm{H}$, $\left.\mathrm{ArCH}_{2} \mathrm{~N}\right), 7.28(\mathrm{~s}, 4 \mathrm{H}, \mathrm{ArH}) .{ }^{13} \mathrm{C}\left\{{ }^{1} \mathrm{H}\right\}-\mathrm{NMR}\left(100 \mathrm{MHz}, \mathrm{CDCl}_{3}\right)$ : $\delta=28.6\left(\mathrm{C}\left(\mathrm{CH}_{3}\right)_{3}\right), 31.4\left(\mathrm{ArC}\left(\mathrm{CH}_{3}\right)_{3}\right), 34.7\left(\mathrm{ArC}\left(\mathrm{CH}_{3}\right)_{3}\right), 37.1$ $\left(\mathrm{SCH}_{2}\right), 41.3\left(\mathrm{NCH}_{3}\right), 45.6\left(\left(\mathrm{NCH}_{2} \mathrm{CH}_{2}\right)_{2} \mathrm{~N}\right), 56.7\left(\left(\mathrm{NCH}_{2} \mathrm{CH}_{2}\right)_{2} \mathrm{~N}\right)$, $\left.61.9\left(\mathrm{ArCH}_{2} \mathrm{~N}\right), 79.5\left(C\left(\mathrm{CH}_{3}\right)_{3}\right), 126.8(\mathrm{ArC}-3,3), 131.4(\mathrm{ArC}-2,2)\right)$, 143.8 (ArC-1), 150.9 (ArC-4), $155.7(C=\mathrm{O})$. This compound was additionally characterized by X-ray crystallography.

Compound $24 \cdot \mathbf{C F}_{3} \mathbf{C O O H}$. To a suspension of $23(250 \mathrm{mg}$, $0.29 \mathrm{mmol})$ in $\mathrm{CH}_{2} \mathrm{Cl}_{2}(0.5 \mathrm{~mL})$ was added trifluoroacetic acid ( $1 \mathrm{~mL}$ ). The resulting clear solution was stirred for $2 \mathrm{~h}$ at r.t., and its $\mathrm{pH}$ was brought to 13 with aqueous $\mathrm{KOH}(5 \mathrm{M})$. The aqueous phase was extracted with $\mathrm{CH}_{2} \mathrm{Cl}_{2}(4 \times 5 \mathrm{~mL})$. The organic fractions were combined and dried with anhydrous $\mathrm{MgSO}_{4}$. Evaporation gave the crude product which was not purified further. Yield: $189 \mathrm{mg}$ (0.28 mmol, 97\%), colorless solid. M.p. 170-172 ${ }^{\circ} \mathrm{C}$. Found: C 63.09, $\mathrm{H}$ 8.82, N 11.43; $\mathrm{C}_{38} \mathrm{H}_{64} \mathrm{~N}_{6} \mathrm{~S}_{2} \mathrm{CF}_{3} \mathrm{CO}_{2} \mathrm{H}$ (783.12) requires: $\mathrm{C}$ 61.35, $\mathrm{H}$ 8.37, $\mathrm{N}$ 10.73. $\mathrm{m} / \mathrm{z}(\mathrm{ESI}+, \mathrm{MeOH}): \mathrm{C}_{38} \mathrm{H}_{64} \mathrm{~N}_{6} \mathrm{~S}_{2}$ (668.46) $\left[\mathrm{M}+\mathrm{H}^{+}\right]^{+}$calcd: 669.47; found 669.47. IR (KBr): $\nu / \mathrm{cm}^{-1}=$ $3441 \mathrm{~s}, 3291 \mathrm{~m} \nu(\mathrm{NH}), 2954 \mathrm{~s}, 2852 \mathrm{~m}, 2780 \mathrm{~s}, 1681 \mathrm{~s} \nu(\mathrm{CO}), 1599 \mathrm{~m}$, $1458 \mathrm{~s}, 1394 \mathrm{w}, 1364 \mathrm{~m}, 1258 \mathrm{~m}, 1227 \mathrm{~m}, 1201 \mathrm{~s}, 1175 \mathrm{~s}, 1131 \mathrm{~s}, 1074$ w, 1048 m, 1022 m, 930 w, 890 w, 832 w, 800 m, 720 m. ${ }^{1}$ H-NMR
(400 MHz, $\left.\mathrm{CDCl}_{3}\right): \delta=1.31\left(\mathrm{~s}, 18 \mathrm{H}, \operatorname{ArC}\left(\mathrm{CH}_{3}\right)_{3}\right), 2.07\left(\mathrm{~s}, 12 \mathrm{H}, \mathrm{NCH}_{3}\right)$, $2.83\left(\mathrm{~m}, 8 \mathrm{H},\left(\mathrm{NCH}_{2} \mathrm{CH}_{2}\right)_{2} \mathrm{~N}\right), 3.05\left(\mathrm{~m}, 8 \mathrm{H},\left(\mathrm{NCH}_{2} \mathrm{CH}_{2}\right)_{2} \mathrm{~N}\right)$, $3.53\left(\mathrm{~s}, 4 \mathrm{H},\left(\mathrm{SCH}_{2}\right)\right), 3.70\left(\mathrm{~s}, 8 \mathrm{H}, \mathrm{ArCH}_{2} \mathrm{~N}\right), 7.21(\mathrm{~s}, 4 \mathrm{H}, \mathrm{Ar}-H)$. $\left.{ }^{13} \mathrm{C}\left\{{ }^{1} \mathrm{H}\right\}-\mathrm{NMR}\left(150 \mathrm{MHz}, 400 \mathrm{~K}, \mathrm{DMSO}-\mathrm{d}_{6}\right): \delta=31.3\left(\mathrm{C} \mathrm{CH}_{3}\right)_{3}\right), 34.5$ $\left(C\left(\mathrm{CH}_{3}\right)_{3}\right), 38.0\left(\mathrm{SCH}_{2}\right), 40.8\left(\mathrm{NCH}_{3}\right), 47.3\left(\left(\mathrm{NCH}_{2} \mathrm{CH}_{2}\right)_{2} \mathrm{~N}\right), 56.2$ $\left(\left(\mathrm{NCH}_{2} \mathrm{CH}_{2}\right)_{2} \mathrm{~N}\right), 63.1\left(\mathrm{ArCH}_{2} \mathrm{~N}\right), 117.8\left(\mathrm{q},{ }^{1} \mathrm{~J}=291 \mathrm{~Hz}, \mathrm{CF}_{3}\right), 129.2$ $(\mathrm{ArC}-3,3), 133.4$ (ArC-2,2), 143.3 (ArC-1), 150.8 (ArC-4), 162.1 $\left(\mathrm{CF}_{3} \mathrm{COO}\right) .{ }^{19} \mathrm{~F}-\mathrm{NMR}\left(376 \mathrm{MHz}, \mathrm{CDCl}_{3}\right):-75.9\left(\mathrm{~s}, \mathrm{CF}_{3}\right)$.

\section{Crystallography}

Suitable single crystals of compounds 8, 15, 17.3DMSO, 19. $2 \mathrm{DMSO} \cdot 2 \mathrm{H}_{2} \mathrm{O}, \mathbf{2 0}$, and 23 were selected and mounted on the tip of a glass fibre using perfluoropolyether oil. The data sets for $8,17 \cdot 3 \mathrm{DMSO}, \mathbf{1 9} \cdot 2 \mathrm{DMSO} \cdot 2 \mathrm{H}_{2} \mathrm{O}$, and 20 were collected at 183(2) K using a STOE IPDS-2 diffractometer, while those for 15 and 23 were collected on a STOE IPDS-1 diffractometer at 213(2) K. Graphite monochromated Mo- $K_{\alpha}$ radiation $(\lambda=0.71073 \AA)$ was used throughout. The data were processed with the programs XAREA. ${ }^{32}$ Selected details of the data collection and refinement are given in Table 1 . The structures were solved by direct methods ${ }^{33}$ and refined by full-matrix least-squares techniques on the basis of all data against $F^{2}$ using SHELXL-97. ${ }^{34}$ PLATON was used to search for higher symmetry. ${ }^{35}$ All non-hydrogen atoms were refined anisotropically, except for those of some disordered solvate molecules. Disorder was modelled using split atom models with restrained $\mathrm{Cl}-\mathrm{O}, \mathrm{O} \cdots \mathrm{O}, \mathrm{C}-\mathrm{C}$, and $\mathrm{C} \cdots \mathrm{C}$ distances using appropriate SADI instructions implemented in the SHELXL software package. Graphics were produced with Ortep3 for Windows and PovRAY.

In the crystal structure of 8 two allyl (N1, C18, C19, C20; C21, $\mathrm{C} 22, \mathrm{C} 23)$ and one ethylene group $(\mathrm{C} 12, \mathrm{C} 13)$ were found to be disordered over two sites. The site occupancies of one allyl and one ethyl group were fixed $(0.74 / 0.26)$. The site occupancies of the other allyl group were refined $(0.55 / 0.45)$. In the crystal structure of 17.3DMSO one DMSO solvate molecule (S5, O15, C57, C58) was found to be heavily disordered and was therefore removed from the structure (and the corresponding $F_{\mathrm{o}}$ ) with the SQuEEzE procedure implemented in the PLATON program suite. Removing the DMSO molecule led to a solvent accessible void of $257 \AA^{3}$, in good agreement with the space needed by one DMSO molecules. The solvate molecules in 19.2DMSO $2 \mathrm{H}_{2} \mathrm{O}$ were also found to be heavily disordered and were therefore removed utilizing the SQUEEZE procedure. This led to solvent accessible voids of $628 \AA^{3}$, attributed to the space needed for two DMSO and two $\mathrm{H}_{2} \mathrm{O}$ molecules.

\section{Results and discussion}

\section{Synthesis}

Scheme 1 depicts the synthetic procedures for compounds $\mathbf{8 , 9}$, and 15-20. The reaction of $\mathbf{1}$ with allylbromide in the presence of $\mathrm{NEt}_{3}$ in ethanol furnished the bicycle 8 in good yields $(>81 \%)$. To prevent overalkylation the reaction was carried out at $0{ }^{\circ} \mathrm{C}$. Under similar conditions, the dimethylated precursor 2 reacted preferentially in the benzylic position providing the 
Table 1 Selected crystallographic data for compounds 8, 15, 17.3DMSO, 19.2DMSO $2 \mathrm{H}_{2} \mathrm{O}, 20$, and 23

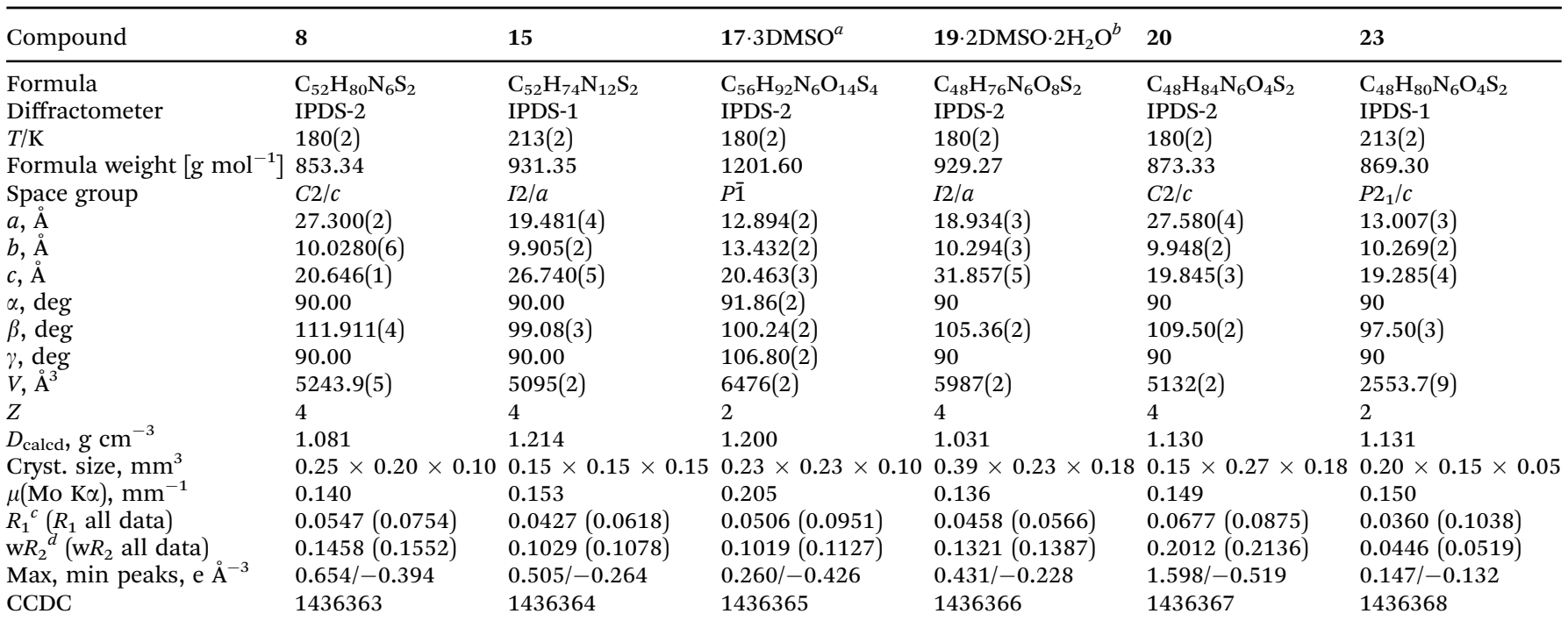

${ }^{a}$ Three DMSO solvate molecules were located from the Fourier map but one was heavily disordered such that it was removed from the structure. ${ }^{b}$ The DMSO and $\mathrm{H}_{2} \mathrm{O}$ solvate molecules were located from the Fourier map but were heavily disordered such that they were all removed from the structure, see Experimental section. ${ }^{c} R_{1}=\Sigma|| F_{\mathrm{o}}|-| F_{\mathrm{c}} \| / \Sigma\left|F_{\mathrm{o}}\right| \cdot{ }^{d} \mathrm{w} R_{2}=\left\{\Sigma\left[\mathrm{w}\left(F_{\mathrm{o}}{ }^{2}-F_{\mathrm{c}}{ }^{2}\right)^{2}\right] / \Sigma\left[\mathrm{w}\left(F_{\mathrm{o}}{ }^{2}\right]\right\}^{1 / 2}\right.$.

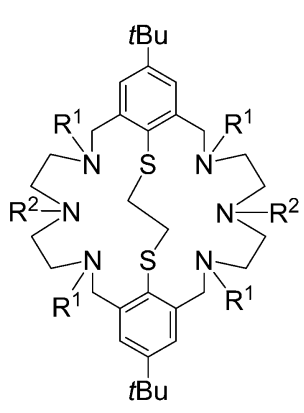

$1\left(R^{1}=R^{2}=H\right)$ $2\left(\mathrm{R}^{1}=\mathrm{H}, \mathrm{R}^{2}=\mathrm{CH}_{3}\right)$

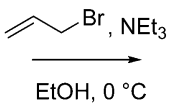

or
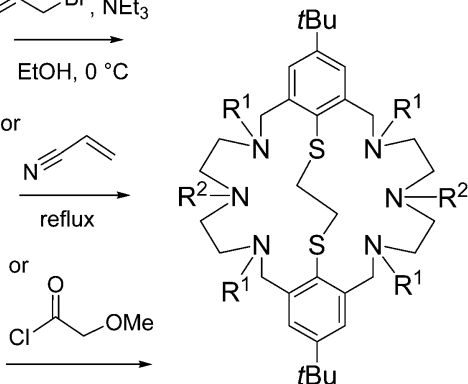

$8\left(\mathrm{R}^{1}=\mathrm{R}^{2}=\mathrm{CH}_{2} \mathrm{CH}=\mathrm{CH}_{2}\right)$ $9\left(\mathrm{R}^{1}=\mathrm{CH}_{2} \mathrm{CH}=\mathrm{CH}_{2}, \mathrm{R}^{2}=\mathrm{CH}_{3}\right)$

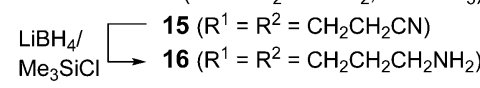

$\mathrm{LiBH}_{4} /-17\left(\mathrm{R}^{1}=\mathrm{R}^{2}=\mathrm{COCH}_{2} \mathrm{OMe}\right)$

$\mathrm{Me}_{3} \mathrm{SiCl} \longrightarrow 18\left(\mathrm{R}^{1}=\mathrm{R}^{2}=\mathrm{CH}_{2} \mathrm{CH}_{2} \mathrm{OMe}\right)$

$\mathrm{LiBH}_{4} /-19\left(\mathrm{R}^{1}=\mathrm{COCH}_{2} \mathrm{OMe}, \mathrm{R}^{2}=\mathrm{CH}_{3}\right)$

$\mathrm{Me}_{3} \mathrm{SiCl} \longrightarrow 20\left(\mathrm{R}^{1}=\mathrm{CH}_{2} \mathrm{CH}_{2} \mathrm{OMe}, \mathrm{R}^{2}=\mathrm{CH}_{3}\right)$
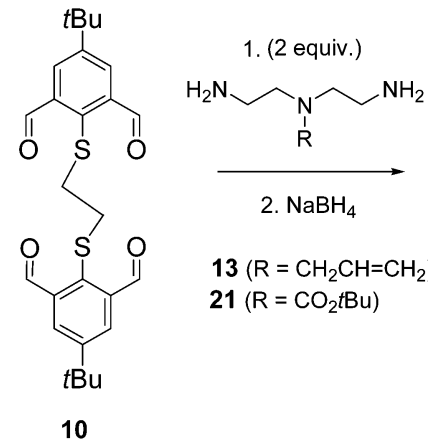

$13\left(\mathrm{R}=\mathrm{CH}_{2} \mathrm{CH}=\mathrm{CH}_{2}\right)$

$21\left(\mathrm{R}=\mathrm{CO}_{2} t \mathrm{Bu}\right)$

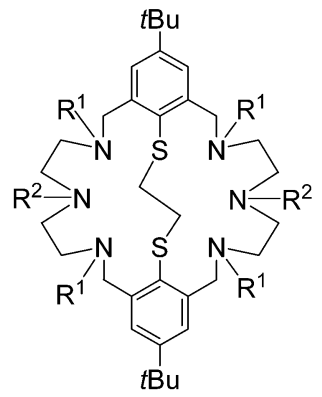

$$
\begin{array}{ll} 
& 14\left(\mathrm{R}^{1}=\mathrm{H}, \mathrm{R}^{2}=\mathrm{CH}_{2} \mathrm{CH}=\mathrm{CH}_{2}\right) \\
\mathrm{CH}_{2} \mathrm{O} / & 22\left(\mathrm{R}^{1}=\mathrm{H}, \mathrm{R}^{2}=\mathrm{CO}_{2} t \mathrm{Bu}\right) \\
\mathrm{NaBH} \mathrm{C}_{3} \mathrm{CN} \longrightarrow & 23\left(\mathrm{R}^{1}=\mathrm{CH}_{3}, \mathrm{R}^{2}=\mathrm{CO}_{2} t \mathrm{Bu}\right) \\
& \downarrow \mathrm{CF}_{3} \mathrm{CO}_{2} \mathrm{H} \\
& 24\left(\mathrm{R}^{1}=\mathrm{CH}_{3}, \mathrm{R}^{2}=\mathrm{H}\right)
\end{array}
$$

Scheme 2 Synthesis of compounds 14 and 22-24.

or $\mathrm{H}_{2}$-RANEY ${ }^{\mathbb{R}}$-Nickel. ${ }^{37-39}$ In our hands, the nitrile 15 failed to react in this fashion. Therefore, an alternative protocol involving reduction with $\mathrm{LiBH}_{4} / \mathrm{Me}_{3} \mathrm{SiCl}$ was employed. ${ }^{40}$ This sequence provided the hexa(3-aminopropylated) macrocycle 16 in moderate to good yields.

The route used for the synthesis of the amino-thioethers with methoxymethyl substituent is depicted in Scheme 1. A reaction sequence similar to that developed for similar $\mathrm{N}_{6} \mathrm{~S}_{2}$-type macrocycles bearing "innocent" alkyl groups was employed. Key-step of this procedure is the acylation of 1 with 2-methoxyacetyl chloride. Thus, in reaction with 2-methoxyacetyl chloride the amide $\mathbf{1 7}$ was generated quantitatively and then reduced to 18 with $\mathrm{LiBH}_{4} / \mathrm{Me}_{3} \mathrm{SiCl}$. As an illustration of the utility of this sequence, the $N, N^{\prime}$-dimethyl derivative 2 was also quantitatively derivatized giving the bicyclic macrocycles 19 and 20, respectively. 


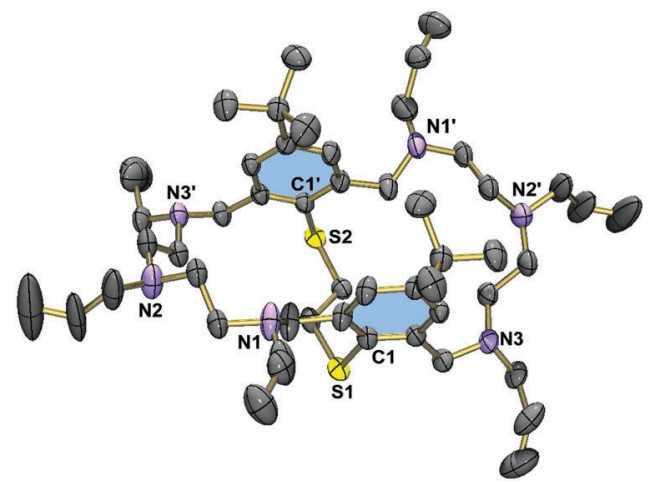

Fig. 2 Molecular structure of $\mathbf{8}$ in the crystal with atomic numbering for key atoms. Thermal ellipsoids are drawn at the 50\% probability level. Hydrogen atoms have been omitted for clarity. Symmetry code used to generate equivalent atoms: $1-x, y, 0.5-z$.

So far only the precursors $\mathbf{1}$ and $\mathbf{2}$ had been utilized for functionalization. In reactions with 1 all six $\mathrm{NH}$ donors are derivatized, while modifications of 2 involved only the benzylic NH donors. We decided to develop a method that allows the selective functionalization of the two central NH donors. In an orienting experiment, the reductive amination of the tetraaldehyde $\mathbf{1}$ with tert-butyl-bis(2-aminoethyl)carbamate $\mathbf{2 1}$ was undertaken. This provided the desired macrocycle 22, albeit in low yield. Having succeeded with the preparation of 22, alkylation of the $\mathrm{N}$-benzyl functions and deprotection of the carbamoyl groups could be examined. Indeed, 22 readily underwent reductive methylation with formaldehyde and $\mathrm{NaBH}_{3} \mathrm{CN}$ to give the tetramethylated derivative 23 in $75 \%$ yields, which was fully characterized including X-ray crystallography. Finally, deprotection of 23 with trifluoroacetic acid gave the desired amine $\mathbf{2 4}$.

All new compounds were characterized by elemental analysis, IR, ${ }^{1} \mathrm{H}$ and ${ }^{13} \mathrm{C}$ NMR spectroscopy. Some compounds were further characterized by X-ray crystallography, in order to study their host-guest chemistry.

\section{Crystal structures}

Fig. 2 displays the molecular structure of the hexaallylated macrocycle 8. The molecule has crystallographically imposed $\mathrm{C}_{2}$ symmetry, and adopts a folded conformation. Unlike in the permethylated derivative $3,{ }^{29}$ the two aromatic rings are essentially coplanar, but are twisted about the $\mathrm{S} 1 \cdots \mathrm{S} 1^{\prime}$ vector (torsional angle $\mathrm{C} 1-\mathrm{S} 1 \cdots \mathrm{S} 1^{\prime}-\mathrm{C} 1 \mathrm{a}=37.8^{\circ}$ ), attributed to steric interactions between the tert-butyl groups. The allyl residues are all oriented away from the cavity. There are no specific intermolecular interactions in 8. The $\mathrm{C}-\mathrm{S}$ bonds are of length $1.783(1) \AA\left(\mathrm{S} 1-\mathrm{C} 1, \mathrm{~S} 1^{\prime}-\mathrm{C}^{\prime}{ }^{\prime}\right)$. Virtually the same distances are seen in $\mathbf{3}$.

Fig. 3 displays the molecular structure of the hexanitrile $\mathbf{1 5 .}$ The macrobicycle adopts a folded conformation, which is similar but not identical to that seen in $\mathbf{8}$. Here, the two phenyl rings plane are bent into the cleft formed by the macrocycle, at an interplanar angle of $19^{\circ}$. The structure is stabilised by two intermolecular $\mathrm{CH} \cdots \pi$ interactions as indicated by relatively short distances between the methylene groups and the aromatic rings $(\mathrm{C} 11 \cdots$ centroid(aromatic ring $)=3.823 \AA$ ) ${ }^{41}$ In contrast to the hexaallylated macrocycle, molecules of $\mathbf{1 5}$ are connected via intermolecular $\mathrm{CH}_{2} \cdots \mathrm{NC}$ interactions $\left(\mathrm{N} 4 \cdots \mathrm{H} 17 \mathrm{~b}^{\prime \prime}=3.013\right.$, $\mathrm{N} 5 \cdots \mathrm{H} 25 \mathrm{~b}^{\prime \prime} 2.517, \mathrm{~N} 6 \cdots \mathrm{H} 19 \mathrm{~b}^{\prime \prime} 2.735 \AA$ A). These interactions lead to a three-dimensional network. The structure of the tetramethoxyethylated aza-thioether 20 is very similar to that of 15 (when neglecting the different $\mathrm{N}$-substituents). However, the tilting of the two aryl rings is not so pronounced $\left(5^{\circ}\right)$ and the C11 $\cdots$ centroid distances are longer at $3.875 \AA$ A.

Hexa(2-methoxyacetylated) macrobicycle 17 crystallizes from DMSO with three solvate molecules. Fig. 4 shows the structure of the macrobicycle, which forms an inclusion complex with a DMSO molecule. The guest molecule is held in place by a $\mathrm{CH} \cdots \pi$ interaction of length $3.823 \AA$ (C11 $\cdots$ centroid(aromatic ring)). The other two DMSO molecules are enclathrated in the voids of the structure. The structure of 17.3DMSO should be compared with that of the tetra(2-methoxyacetylated) derivative 19.2DMSO $\cdot 2 \mathrm{H}_{2} \mathrm{O}$ (Fig. 4, right). This compound crystallizes also

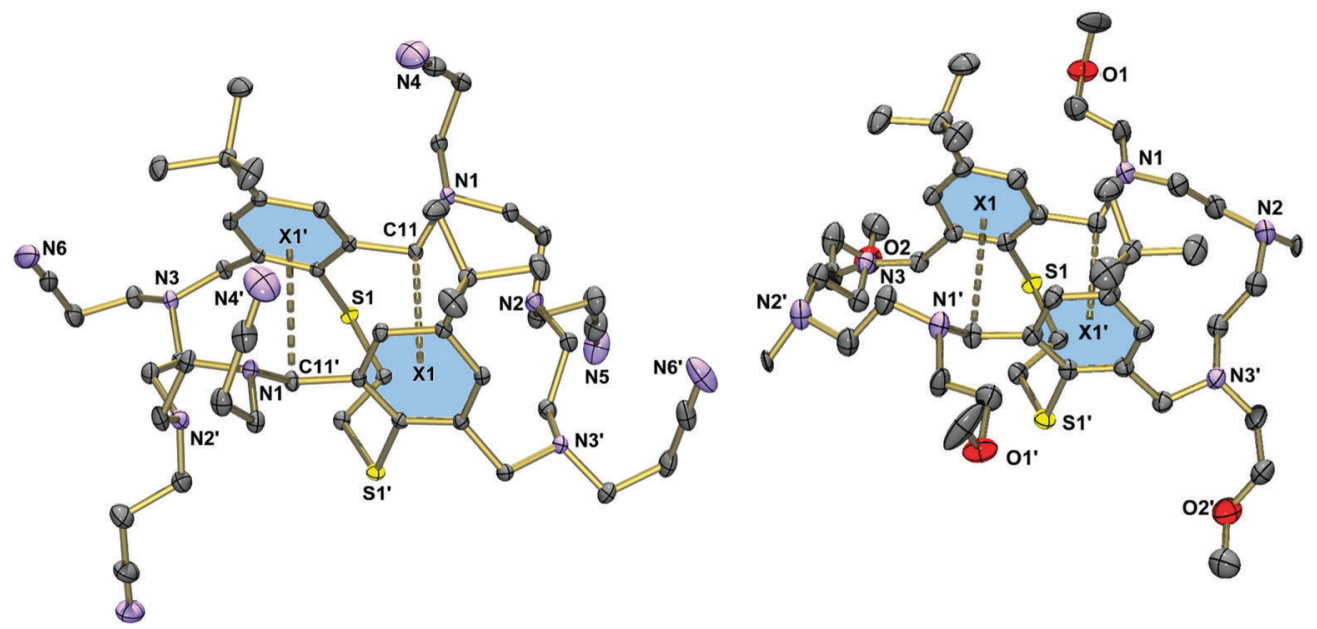

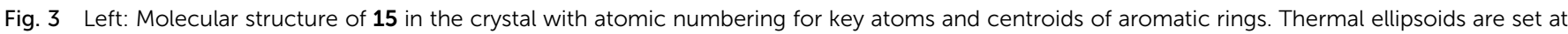

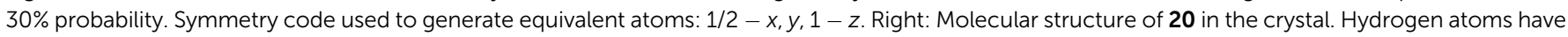
been omitted for clarity. Symmetry code: $-x, y, 1 / 2-z . \mathrm{CH} \cdots \pi$ interactions indicated by dashed lines. 

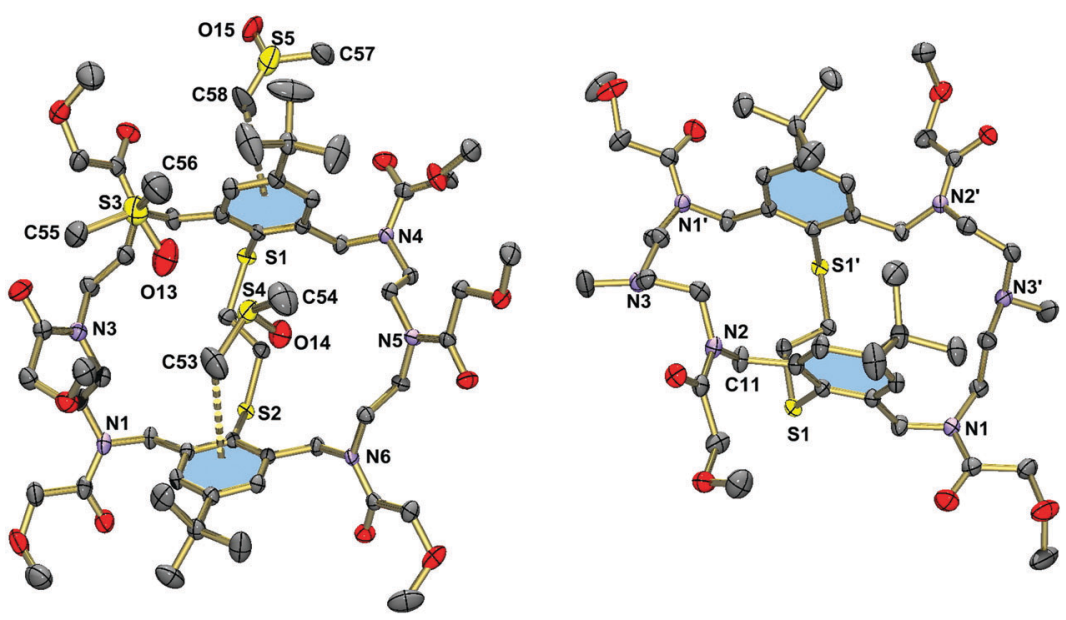

Fig. 4 Left: Molecular structure of 17.3DMSO in the crystal. The atom N3 is obscured by a DMSO molecule (O(13)S(3)C(56)C(57)). The DMSO molecule $\mathrm{O}(15) \mathrm{S}(5) \mathrm{C}(58) \mathrm{C}(59)$ is disordered over two positions. Only one orientation is displayed. Right: Molecular structure of $19 \cdot 2 \mathrm{DMSO} \cdot 2 \mathrm{H}_{2} \mathrm{O}$ in the $\mathrm{crystal}$. Thermal ellipsoids are set at 50\% probability. Hydrogen atoms have been omitted for clarity.

with solvate molecules, but does not form an inclusion complex. The two phenyl rings in $\mathbf{1 9}$ are coplanar as in the hexanitrile 15. However, the distance between the two best planes through the benzene rings is much larger at $5.087 \AA$. As a consequence, the phenyl rings are not involved in intermolecular $\mathrm{CH} \cdots \pi$ interactions with the adjacent benzyl group $($ C11 ‥centroid(aromatic ring $)=4.802 \AA)$. Clearly, removal of two methoxyacetyl residues exerts more conformational flexibility on the macrocycle.

Fig. 5 displays the structure of the protected macrobicycle 23, which has crystallographically imposed inversion symmetry. Unlike in the above structures, the thioether adopts a stepped conformation, presumably a consequence of the steric requirements of the $\mathrm{N}$-carbamate groups. As a consequence, the macrocycles are engaged in intermolecular $\mathrm{CH} \cdots \pi$ interactions. The corresponding

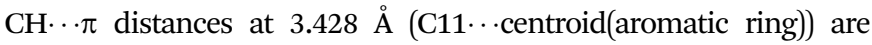
significantly shorter than in $\mathbf{1 5}$ or $\mathbf{2 0}$. This compound crystallizes without guest molecules.

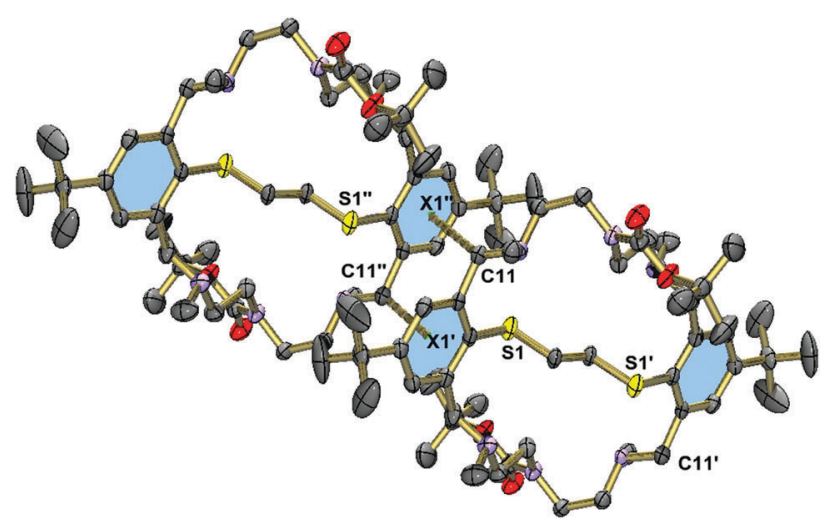

Fig. 5 Molecular structure of compound $\mathbf{2 3}$ in the crystal showing the intermolecular $\mathrm{CH} \cdots \pi$ interactions. Hydrogen atoms are omitted for clarity. Selected bond lengths [Å]: C1-S1 1.789(3) A. Symmetry codes: $-x, 1-y,-z\left({ }^{\prime}\right) ;-x, 2-y,-z\left(^{\prime \prime}\right)$.

\section{Conclusion}

Overall, a short and efficient protocol for the functionalization of bicyclic aza-thioethers has been described. All six secondary amine functions of the parent macrobicycles $\mathbf{1}$ and $\mathbf{2}$ are readily alkylated without overalkylation and without affecting the masked thiolate functions. The protocol is also feasible for the synthesis of macrobicycles with different alkyl groups on the benzylic and central nitrogen atoms of the linking diethylene triamine units, such that these derivatives are also now available. Six of the twelve new compounds were obtained in crystalline form, such that their molecular structures could be determined. In the solid state the macrobicycles can adopt a stepped or a folded conformation. The structures appear to be primarily governed by inter- and intramolecular $\mathrm{CH}_{2} \cdots \pi$ interactions (involving the benzylic methylene groups and the aromatic rings) rather due to steric effects played by the $N$ alkyl functions. The observation that DMSO, which is a good CH donor, can form an inclusion complex held in place by a $\mathrm{CH}_{3} \cdots \pi$ interaction would be consistent with this in view.

\section{Acknowledgements}

We are thankful to Prof. Dr H. Krautscheid for providing facilities for X-ray crystallographic measurements. This work was supported by the Deutsche Forschungsgemeinschaft (DFG Research unit 1154, "Towards Molecular Spintronics").

\section{References}

1 E. C. Constable, Coordination Chemistry of Macrocyclic Compounds, Oxford University Press, Oxford, 1999, ch. 5.

2 L. Lindoy, The chemistry of macrocyclic ligand complexes, Cambridge University Press, Cambridge, 1990, ch. 6.

3 D. H. Busch, Acc. Chem. Res., 1978, 11, 392-400. 
4 F. A. Cotton, G. Wilkinson, C. A. Murillo and M. Bochmann, Advanced Inorganic Chemistry, John Wiley \& Sons, Weinheim, 6th edn, 1999.

5 (a) M. Schröder and V. Lippolis, in Macrocyclic Chemistry, Current Trends and Future Perspectives, ed. K. Gloe, Springer, Dordrecht, The Netherlands, 2005, ch. 5, p. 67; (b) L. Tei, A. Bencini, A. J. Blake, V. Lippolis, A. Perra, B. Valtancoli, C. Wilson and M. Schröder, Dalton Trans., 2004, 1934-1944.

6 (a) K. P. Wainwright, Coord. Chem. Rev., 1997, 166, 35-90; (b) K. P. Wainwright, Adv. Inorg. Chem., 2001, 52, 293-334.

7 P. V. Bernhardt and G. A. Lawrence, Coord. Chem. Rev., 1990, 104, 297-343.

8 D. S. C. Black and I. A. McLean, Chem. Commun., 1968, 1004-1006.

9 B. Dietrich, J. M. Lehn and J. P. Sauvage, Chem. Commun., 1970, 1055-1056.

10 (a) U. Kallert and R. Mattes, Inorg. Chim. Acta, 1991, 180, 263-269; (b) K. Wasielewski and R. Mattes, Z. Anorg. Allg. Chem., 1993, 619, 158-162.

11 I. Bertini, L. Sacconi and G. P. Speroni, Inorg. Chem., 1972, 11, 1323-1326.

12 R. C. Coombes, J.-P. Costes and D. E. Fenton, Inorg. Chim. Acta, 1983, 77, 173-174.

13 D. A. Nation, M. R. Taylor and K. P. Wainwright, J. Chem. Soc., Dalton Trans., 1992, 1557-1562.

14 L. F. Lindoy and R. J. Smith, Inorg. Chem., 1981, 20, 1314-1316. 15 H. Koyama and I. Murase, Bull. Chem. Soc. Jpn., 1977, 50, 895-897.

16 (a) T. Yamamura, M. Tadokora and R. Kuroda, Chem. Lett., 1989, 1245-1246; (b) T. Yamamura, M. Tadokoro, K. Tanaka and R. Kuroda, Bull. Chem. Soc. Jpn., 1984, 66, 1984-1990.

17 J. W. L. Martin, G. J. Organ, K. P. Wainwright, K. D. V. Weerasuria, A. C. Willis and S. B. Wild, Inorg. Chem., 1987, 26, 2963-2968.

18 C. J. Hinshaw, G. Peng, R. Singh, J. T. Spence, J. H. Enemark, M. Bruck, J. Kristowski, S. L. Merbs, R. B. Ortega and P. A. Wexler, Inorg. Chem., 1989, 28, 4483-4491.

19 Y. Sun, C. S. Cutler, A. E. Martell and M. J. Welch, Tetrahedron, 1999, 55, 5733-5740.

20 N. Ehlers, D. Funkemeier and R. Mattes, Z. Anorg. Allg. Chem., 1994, 620, 796-800.

21 M. W. A. Steenland, P. Westbroek, I. Dierck, G. G. Herman, W. Lippens, E. Temmerman and A. M. Goeminne, Polyhedron, 1999, 18, 3417-3424.

22 I. M. Vasilescu, D. J. Bray, J. K. Clegg, L. F. Lindoy, G. V. Meehan and G. Wei, Dalton Trans., 2006, 5115-5117.

23 (a) M. C. Aragoni, M. Arca, A. Bencini, C. Caltagirone, A. Garau, F. Isaia, M. E. Light, V. Lippolis, C. Lodeiro, M. Mameli, R. Montis, M. C. Mostallino, A. Pintus and S. Puccioni, Dalton Trans., 2013, 42, 14516-14530; (b) K. Zargoosh, M. Shamsipur, M. Hosseini, C. Caltagirone and V. Lippolis, J. Lumin., 2012, 132, 2126-2129; (c) M. Mameli, V. Lippolis, C. Caltagirone, J. L. Capelo, O. N. Faza and C. Lodeiro, Inorg. Chem., 2010, 49,
8276-8286; (d) M. Mameli, M. C. Aragoni, M. Arca, M. Atzori, A. Bencini, C. Bazzicalupi, A. J. Blake, C. Caltagirone, F. A. Devillanova, A. Garau, M. B. Hursthouse, F. Isaia, V. Lippolis and B. Valtancoli, Inorg. Chem., 2009, 48, 9236-9249; (e) M. C. Aragoni, M. Arca, A. Bencini, A. J. Blake, C. Caltagirone, A. Decortes, F. Demartin, F. A. Devillanova, E. Faggi, L. S. Dolci, A. Garau, F. Isaia, V. Lippolis, L. Prodi, C. Wilson, B. Valtancoli and N. Zaccheroni, Dalton Trans., 2005, 2994-3004; $(f)$ C. Caltagirone, A. Bencini, F. Demartin, F. A. Devillanova, A. Garau, F. Isaia, V. Lippolis, P. Mariani, U. Papke, L. Tei and G. Verani, Dalton Trans., 2003, 901-909.

24 (a) B. Kersting and G. Steinfeld, Chem. Commun., 2001, 1376-1377; (b) M. H. Klingele, G. Steinfeld and B. Kersting, Z. Naturforsch., 2001, 56b, 901-907; (c) M. H. Klingele and B. Kersting, Z. Naturforsch., 2001, 56b, 437-439.

25 M. Gressenbuch and B. Kersting, Eur. J. Inorg. Chem., 2007, 90-102.

26 M. Gressenbuch, V. Lozan, G. Steinfeld and B. Kersting, Eur. J. Inorg. Chem., 2005, 2223-2234.

27 T. Gregor, C. F. Weise, V. Lozan and B. Kersting, Synthesis, 2007, 3706-3712.

28 M. Gressenbuch and B. Kersting, Dalton Trans., 2009, 5281-5283.

29 G. Siedle and B. Kersting, Z. Anorg. Allg. Chem., 2003, 629, 2083-2090.

30 C. Y. Ng, R. J. Motekaitis and A. E. Martell, Inorg. Chem., 1979, 18, 2982-2986.

31 (a) M. P. Hay, F. B. Pruijn, S. A. Gamage, H. D. S. Liyanage, M. S. Kovacs, A. V. Patterson, W. R; Wilson, J. M. Brown and W. A. Denny, J. Med. Chem., 2004, 47, 475-488; (b) M. A. Ilies, W. A. Seitz, B. H. Johnson, E. L. Ezell, A. L. Miller, E. B. Thompson and A. T. Balaban, J. Med. Chem., 2006, 49, 3872-3887.

32 Stoe \& Cie, $X$-AREA and $X$-RED 32; V1.35, Stoe \& Cie, Darmstadt, Germany, 2006.

33 G. M. Sheldrick, Acta Crystallogr., Sect. A: Found. Crystallogr., 1990, 46, 467-473.

34 G. M. Sheldrick, SHELXL-97, Computer program for crystal structure refinement, University of Göttingen, Göttingen, Germany, 1997.

35 A. L. Spek, PLATON - A Multipurpose Crystallographic Tool, Utrecht University, Utrecht, The Netherlands, 2000.

36 K. P. Wrainwright, J. Chem. Soc., Dalton Trans., 1980, 2117-2120. 37 K. P. Wrainwright, J. Chem. Soc., Dalton Trans., 1983, 1149-1152. 38 R. J. Bergeron and J. R. Garlich, Synthesis, 1984, 782-784.

39 C. Gros, H. Chollet, A. K. Mishra and R. Guilard, Synth. Commun., 1996, 26, 35-47.

40 A. Giannis and K. Sandhoff, Angew. Chem., 1989, 101, 220-222 (Angew. Chem., Int. Ed. Engl., 1989, 28, 218-220).

41 E. A. Meyer, R. K. Castellano and F. Diederich, Angew. Chem., 2003, 115, 1244-1287 (Angew. Chem., Int. Ed., 2003, 42, 1210-1250). 